TRANSACTIONS OF THE

AMERICAN MATHEMATICAL SOCIETY

Volume 356, Number 12, Pages 4787-4809

S 0002-9947(04)03704-3

Article electronically published on June 25, 2004

\title{
SECOND ORDER PARABOLIC EQUATIONS IN BANACH SPACES WITH DYNAMIC BOUNDARY CONDITIONS
}

\author{
TI-JUN XIAO AND JIN LIANG
}

\begin{abstract}
In this paper, we exhibit a unified treatment of the mixed initial boundary value problem for second order (in time) parabolic linear differential equations in Banach spaces, whose boundary conditions are of a dynamical nature. Results regarding existence, uniqueness, continuous dependence (on initial data) and regularity of classical and strict solutions are established. Moreover, several examples are given as samples for possible applications.
\end{abstract}

\section{INTRODUCTION}

Of concern is the inhomogeneous complete second order differential equation

$$
u^{\prime \prime}(t)+A u(t)+B u^{\prime}(t)=f(t), \quad t>0,
$$

in a Banach space $E$, where $A$ and $B$ are linear operators in $E$, and $f$ an $E$-valued function. The Cauchy problem for (1.1) has been extensively studied since the end of 1950s (see H. O. Fattorini [9] 10] and T. J. Xiao and J. Liang 24 25] for surveys).

In this paper, we consider a mixed initial boundary value problem for (1.1), in which besides the usual initial condition

$$
u(0)=u_{0}, \quad u^{\prime}(0)=u_{1},
$$

there is also a boundary condition given by

$$
x^{\prime \prime}(t)+A_{1} x(t)+B_{1} x^{\prime}(t)=G_{0} u(t)+G_{1} u^{\prime}(t)+g(t), \quad t>0 .
$$

Here $x(\cdot)$ stands for the boundary value of the state function $u(\cdot)$, these two functions being connected by a linear boundary operator $P$ (from $\mathcal{D}(A)$ to another Banach space $X$ ),

$$
x(t)=P u(t), \quad t>0
$$

$A_{1}$ and $B_{1}$ are linear operators in $X, g$ an $X$-valued function, and $G_{i}(i=0,1)$ are linear operators (feedback operators) from $\mathcal{D}\left(G_{i}\right) \subset E$ to $X$. The boundary condition (1.3) is of a dynamic nature, for which we initially have

$$
x(0)=x_{0}, \quad x^{\prime}(0)=x_{1} .
$$

Received by the editors June 24, 2002.

2000 Mathematics Subject Classification. Primary 34G10, 47D06, 35G10.

Key words and phrases. Differential equations in Banach spaces, second order (in time), parabolic type, dynamic boundary conditions, strict and classical solutions.

The first author acknowledges support from the Alexander-von-Humboldt Foundation and from CAS and NSFC. The second author acknowledges support from the Max-Planck Society and from CAS and EMC. 
Dynamic boundary conditions occur in diverse practical problems, for instance, in those modelling the dynamic vibrations of linear viscoelastic rods and beams with tip masses attached at their free ends; see, e.g., [2, 4, 21]. The study of evolution equations with dynamic boundary conditions from the mathematical point of view dates back to 1961, when J. L. Lions [18, p. 117, 118] treated such equations and gave weak solutions by means of the variational method. Since then, this issue has been investigated to a large extent (see, e.g., 5, 6, 8, 11, 12, 13, 15, 16, 17, 18 22 and references therein). While most of the previous research concerns the case of first order in time, there has been little regarding the second order (in time) case. It seems nontrivial or impractical, as far as dynamic boundary conditions are concerned, to get solutions for a second order (in time) problem by reducing it to a first order one, especially to obtain strong solutions with high time regularity and spatial regularity (cf. [1, Theorem 2.1 and Remark 4.2]). In the present paper, we shall deal with the second order problem (11.1) - (1.5) in a direct way, without reduction. This approach will yield strong solutions with desirable regularity, as well as build up theorems of a general nature.

To begin, write

$$
\begin{aligned}
& \mathbb{A}:=\left(\begin{array}{cc}
A & 0 \\
-G_{0} & A_{1}
\end{array}\right), \quad \mathcal{D}(\mathbb{A}):=\left\{\left(\begin{array}{l}
u \\
x
\end{array}\right) \in\left(\mathcal{D}(A) \cap \mathcal{D}\left(G_{0}\right)\right) \times \mathcal{D}\left(A_{1}\right) ; \quad x=P u\right\}, \\
& \mathbb{B}:=\left(\begin{array}{cc}
B & 0 \\
-G_{1} & B_{1}
\end{array}\right), \quad \mathcal{D}(\mathbb{B}):=\left(\mathcal{D}(B) \cap \mathcal{D}\left(G_{1}\right)\right) \times \mathcal{D}\left(B_{1}\right), \\
& y(t):=\left(\begin{array}{c}
u(t) \\
x(t)
\end{array}\right), \quad h(t):=\left(\begin{array}{l}
f(t) \\
g(t)
\end{array}\right), \quad y_{0}:=\left(\begin{array}{l}
u_{0} \\
x_{0}
\end{array}\right), \quad y_{1}:=\left(\begin{array}{l}
u_{1} \\
x_{1}
\end{array}\right) .
\end{aligned}
$$

Then, problem (1.1) - (1.5) is converted into an abstract Cauchy problem in the product space $\mathbf{E}:=E \times X$ :

$$
\left\{\begin{array}{l}
y^{\prime \prime}(t)+\mathbb{A} y(t)+\mathbb{B} y^{\prime}(t)=h(t), \quad t>0, \\
y(0)=y_{0}, y^{\prime}(0)=y_{1} .
\end{array}\right.
$$

How can one deal with this problem involving two operator matrices? We shall present some ideas about it. This paper is confined to equations of parabolic type, and those of hyperbolic type will be considered in a forthcoming paper.

In order to carry out our strategy, we still need to introduce another boundary operator $P_{1}$, a linear operator from $\mathcal{D}(B)$ to the quotient space $X / X_{0}\left(X_{0}\right.$ is a closed linear subspace of $X$ ). The $P_{1}$ can be chosen flexibly in applications (see Examples 4.14 .3 and 4.5), such that the relation

$$
x^{\prime}(t) \in P_{1} u^{\prime}(t), \quad t>0,
$$

is implied by (1.1), (1.3) and (1.4). The simplest $P_{1}$ is in the case of $X_{0}=X$.

For the two operators $A$ and $B$ in the state space $E$, we define

$$
A_{0}:=\left.A\right|_{\text {ker } P}, \quad B_{0}:=\left.B\right|_{\text {ker } P_{1}} .
$$

Then the elements in the domains of $A_{0}$ and $B_{0}$ have zero boundary values in some sense. A condition of parabolic type will be given on the operator pair $\left(A_{0}, B_{0}\right)$ (also on $\left(A_{1}, B_{1}\right)$ ), which is easy to verify in concrete situations. Moreover, for equations (1.1) and (1.3), we regard $A, B, A_{1}$, and $B_{1}$ as principal operators to 
which $G_{0}$ and $G_{1}$ are subordinated. For a wider applicability, we take four more perturbing (linear) operators into consideration:

$$
\begin{aligned}
& \widetilde{A}: \mathcal{D}(\widetilde{A}) \subset E \rightarrow E, \quad \widetilde{B}: \mathcal{D}(\widetilde{B}) \subset E \rightarrow E, \\
& \widetilde{A}_{1}: \mathcal{D}\left(\widetilde{A}_{1}\right) \subset X \rightarrow X, \quad \widetilde{B}_{1}: \mathcal{D}\left(\widetilde{B}_{1}\right) \subset X \rightarrow X .
\end{aligned}
$$

Thus, we shall actually study

$$
\left\{\begin{array}{l}
y^{\prime \prime}(t)+(\mathbf{A}+\widetilde{\mathbf{A}}) y(t)+(\mathbf{B}+\widetilde{\mathbf{B}}) y^{\prime}(t)=h(t), \quad t>0 \\
y(0)=y_{0}, y^{\prime}(0)=y_{1}
\end{array}\right.
$$

in space $\mathbf{E}$, with the main operator matrices $\mathbf{A}, \mathbf{B}$ and the perturbing operators $\widetilde{\mathbf{A}}$, $\widetilde{\mathbf{B}}$ defined as follows:

$$
\begin{aligned}
\mathbf{A} & :=\left(\begin{array}{cc}
A & 0 \\
0 & A_{1}
\end{array}\right), \quad \mathcal{D}(\mathbf{A}):=\left\{\left(\begin{array}{l}
u \\
x
\end{array}\right) \in \mathcal{D}(A) \times \mathcal{D}\left(A_{1}\right) ; \quad x=P u\right\}, \\
\mathbf{B} & :=\left(\begin{array}{cc}
B & 0 \\
0 & B_{1}
\end{array}\right), \quad \mathcal{D}(\mathbf{B}):=\left\{\left(\begin{array}{l}
u \\
x
\end{array}\right) \in \mathcal{D}(B) \times \mathcal{D}\left(B_{1}\right) ; \quad x \in P_{1} u\right\} . \\
\widetilde{\mathbf{A}} & :=\left(\begin{array}{cc}
\widetilde{A} & 0 \\
-G_{0} & \widetilde{A}_{1}
\end{array}\right), \quad \widetilde{\mathbf{B}}:=\left(\begin{array}{cc}
\widetilde{B} & 0 \\
-G_{1} & \widetilde{B}_{1}
\end{array}\right) .
\end{aligned}
$$

In Section 2, we shall show under suitable conditions that the operator pair $(\mathbf{A}+\widetilde{\mathbf{A}}, \mathbf{B}+\widetilde{\mathbf{B}})$ possesses certain parabolicity (Theorem 2.3), and then construct an operator function $\widetilde{\mathbf{S}}(\cdot)$ (a fundamental solution operator of (1.8) ) having a holomorphic extension to a sector $\Sigma_{\theta}\left(\theta \in\left(0, \frac{\pi}{2}\right]\right)$ and satisfying various nice properties (Theorem 2.4). Making use of this, we will formulate and prove, in Section 3, our main theorem (Theorem 3.3) with regard to the existence and uniqueness of classical and strict solutions for (1.8), also continuous dependence (on initial data) and regularity of the solutions. Finally, in Section 4 we shall exhibit three applications of our theorems to damped beam and plate-like equations with dynamic boundary conditions.

Notation. For Banach spaces $E$ and $X, \mathcal{L}(E, X)$ is the space of all bounded linear operators from $E$ into $X$. The space $\mathcal{L}(E, E)$ is abbreviated to $\mathcal{L}(E)$. For a linear operator $A$ in $E, \mathcal{D}(A)$, $\operatorname{ker} A$, and $\rho(A)$ stands for its domain, kernel, and resolvent set, respectively. The operator $\left.A\right|_{Z}$ means the restriction of $A$ to a space Z. $C^{\alpha}([0, T] ; E)(\alpha \in(0,1))$ is the Banach space of Hölder continuous functions $q:[0, T] \rightarrow E$ with exponent $\alpha$ and norm given by

$$
\sup _{0 \leq t \leq T}\|q(t)\|_{E}+\sup _{0 \leq s<t \leq T}(t-s)^{-\alpha}\|q(t)-q(s)\|_{E} .
$$

Write

$$
\begin{aligned}
& \Sigma_{\theta}:=\{\lambda \in \mathbf{C} ; \quad \lambda \neq 0,|\arg \lambda|<\theta\}, \quad \theta \in(0, \pi], \\
& R_{i}(\lambda):=\left(\lambda^{2}+A_{i}+\lambda B_{i}\right)^{-1}, \quad i=0,1, \quad \lambda \in \mathbf{C}, \\
& \widetilde{\mathbf{R}}(\lambda):=\left(\lambda^{2}+(\mathbf{A}+\widetilde{\mathbf{A}})+\lambda(\mathbf{B}+\widetilde{\mathbf{B}})\right)^{-1}, \quad \lambda \in \mathbf{C},
\end{aligned}
$$

if the inverse operators exist, and

$$
\rho\left(A_{0}, B_{0}\right):=\left\{\lambda \in \mathbf{C} ; R_{0}(\lambda) \text { exists and belongs to } \mathcal{L}(E)\right\} .
$$


By $[\mathcal{D}(A)]$ we denote the space $\mathcal{D}(A)$ equipped with the graph norm, $[\mathcal{D}(A)]_{P}$ the space $\mathcal{D}(A)$ with the norm

$$
\|u\|_{A, P}:=\|u\|+\|A u\|+\|P u\|,
$$

$[\mathcal{D}(B)]_{P_{1}}$ the space $\mathcal{D}(B)$ with the norm

$$
\|u\|_{B, P_{1}}:=\|u\|+\|B u\|+\left\|P_{1} u\right\|_{X / X_{0}},
$$

$[\mathcal{D}(A) \cap \mathcal{D}(B)]$ the space $\mathcal{D}(A) \cap \mathcal{D}(B)$ with the norm

$$
\|u\|_{A, B}:=\|u\|+\|A u\|+\|B u\|,
$$

and $[\mathcal{D}(A) \cap \mathcal{D}(B)]_{P}$ the space $\mathcal{D}(A) \cap \mathcal{D}(B)$ with the norm

$$
\|u\|_{A, B, P}:=\|u\|+\|A u\|+\|B u\|+\|P u\| .
$$

\section{Parabolicity}

We first give some basic properties of the operators $A, B$ and $P$.

Lemma 2.1. Suppose that the following $\left(\mathrm{H}_{1}\right)$ is satisfied.

$\left(\mathrm{H}_{1}\right)[\mathcal{D}(A)]_{P}$ and $[\mathcal{D}(B)]_{P_{1}}$ are complete, $P(\mathcal{D}(A) \cap \mathcal{D}(B))=X$, and $P u \in P_{1} u$ for any $u \in \mathcal{D}(A) \cap \mathcal{D}(B)$.

Then

(1) The space $[\mathcal{D}(A) \cap \mathcal{D}(B)]_{P}$ is complete.

(2) If $\lambda \in \rho\left(A_{0}, B_{0}\right), \lambda \neq 0$, then we have that $\left.P\right|_{\operatorname{ker}\left(\lambda^{2}+A+\lambda B\right)}$ is a bijection of $\operatorname{ker}\left(\lambda^{2}+A+\lambda B\right)$ onto $X$, and

$$
D_{\lambda}:=\left(\left.P\right|_{\operatorname{ker}\left(\lambda^{2}+A+\lambda B\right)}\right)^{-1}
$$

is bounded from $X$ to $\left(\operatorname{ker}\left(\lambda^{2}+A+\lambda B\right),\|\cdot\|_{A, B, P}\right)$.

(3) For every $\lambda, \mu \in \rho\left(A_{0}, B_{0}\right)$ with $\lambda, \mu \neq 0$,

$$
D_{\lambda}=D_{\mu}+(\mu-\lambda) R_{0}(\lambda)(\mu+\lambda+B) D_{\mu} .
$$

Proof. (1) Suppose that $\left\{u_{n}\right\}_{n \in N}$ is a Cauchy sequence in $[\mathcal{D}(A) \cap \mathcal{D}(B)]_{P}$. Then it is easy to see that $\left\{u_{n}\right\}_{n \in N}$ is a Cauchy sequence in $[\mathcal{D}(A)]_{P}$. So there exists $u \in \mathcal{D}(A)$ such that

$$
u_{n} \rightarrow u, \quad A u_{n} \rightarrow A u, \quad P u_{n} \rightarrow P u, \text { as } n \rightarrow \infty .
$$

Moreover, $\left\{u_{n}\right\}_{n \in N}$ is also a Cauchy sequence in $[\mathcal{D}(B)]_{P_{1}}$, because of

$$
\left\|P_{1} u_{n}\right\|_{X / X_{0}} \leq\left\|P u_{n}\right\|
$$

by $\left(\mathrm{H}_{1}\right)$; therefore there is $v \in \mathcal{D}(B)$ such that

$$
\lim _{n \rightarrow \infty} u_{n}=v, \quad \lim _{n \rightarrow \infty} B u_{n}=B v .
$$

Combining (2.2) and (2.3) shows that $u=v$, and so

$$
u \in \mathcal{D}(B), \quad \lim _{n \rightarrow \infty} B u_{n}=B u .
$$

This verifies the completeness of $[\mathcal{D}(A) \cap \mathcal{D}(B)]_{P}$.

(2) Assume that $u, v \in \operatorname{ker}\left(\lambda^{2}+A+\lambda B\right)$, with $P u=P v$. Then

$$
\left(\lambda^{2}+A+\lambda B\right)(u-v)=0 \quad \text { and } \quad P(u-v)=0,
$$


which implies $P_{1}(u-v)=0$. Therefore $u-v \in \mathcal{D}\left(A_{0}\right) \cap \mathcal{D}\left(B_{0}\right)$ by the definitions of $A_{0}$ and $B_{0}$. Thus we have

$$
\left(\lambda^{2}+A_{0}+\lambda B_{0}\right)(u-v)=0 .
$$

This yields that $u-v=0$ since $\lambda \in \rho\left(A_{0}, B_{0}\right)$. Hence $\left.P\right|_{\operatorname{ker}\left(\lambda^{2}+A+\lambda B\right)}$ is injective. Next take $x \in X$. Then there is $u \in \mathcal{D}(A) \cap \mathcal{D}(B)$ such that $P u=x$, by $\left(\mathrm{H}_{1}\right)$. Put

$$
v_{1}=R_{0}(\lambda)\left(\lambda^{2}+A+\lambda B\right) u, \quad v_{2}=u-v_{1} .
$$

We see easily that $v_{1} \in \mathcal{D}\left(A_{0}\right)$ and $\left(\lambda^{2}+A+\lambda B\right) v_{2}=0$. So

$$
P v_{1}=0, \quad P v_{2}=P u-P v_{1}=x,
$$

and $v_{2} \in \operatorname{ker}\left(\lambda^{2}+A+\lambda B\right)$. This indicates that $\left.P\right|_{\operatorname{ker}\left(\lambda^{2}+A+\lambda B\right)}$ is surjective. Finally, we observe that $\left(\operatorname{ker}\left(\lambda^{2}+A+\lambda B\right),\|u\|_{A, B, P}\right)$ is a Banach space in view of (1), and $\left.P\right|_{\operatorname{ker}\left(\lambda^{2}+A+\lambda B\right)}$ is a bounded linear operator from $\left(\operatorname{ker}\left(\lambda^{2}+A+\lambda B\right),\|\cdot\|_{A, B, P}\right)$ onto $X$. So an appeal to the open mapping theorem gives the boundedness of $D_{\lambda}$.

(3) Write

$$
Q=\left[I+(\mu-\lambda) R_{0}(\lambda)(\mu+\lambda+B)\right] D_{\mu} .
$$

Then for each $x \in X$,

$$
\begin{aligned}
\left(\lambda^{2}+A+\lambda B\right) Q x & =\left[\left(\lambda^{2}+A+\lambda B\right)+\mu^{2}-\lambda^{2}+(\mu-\lambda) B\right] D_{\mu} x \\
& =\left(\mu^{2}+A+\mu B\right) D_{\mu} x=0,
\end{aligned}
$$

since $D_{\mu} x \in \operatorname{ker}\left(\mu^{2}+A+\mu B\right)$. Thus we see $\operatorname{Range}(Q) \subset \operatorname{ker}\left(\lambda^{2}+A+\lambda B\right)$. Moreover, we have $P Q=P D_{\mu}=I$, noting $P R_{0}(\lambda)=0$. Therefore, we deduce $Q=D_{\lambda}$ as claimed. The proof is then complete.

The following is the hypotheses of parabolic type on $A_{0}, B_{0}$ (see (1.7)) and on $A_{1}, B_{1}$.

$\left(\mathrm{H}_{2}\right)$ The operators $A_{0}$ and $B_{0}$ are closed, and for each $\varphi \in(0, \theta)\left(\theta \in\left(0, \frac{\pi}{2}\right]\right)$, there exist $M_{\varphi}, \omega_{\varphi}>0$ such that

$$
\left\|\lambda R_{0}(\lambda)\right\|,\left\|\lambda^{-1} A_{0} R_{0}(\lambda)\right\| \leq M_{\varphi}|\lambda|^{-1}, \quad \lambda \in \omega_{\varphi}+\Sigma_{\frac{\pi}{2}+\varphi} .
$$

$\left(\mathrm{H}_{3}\right)$ The operators $A_{1}$ and $B_{1}$ are closed, and for each $\varphi \in(0, \theta)\left(\theta \in\left(0, \frac{\pi}{2}\right]\right)$, there exist $M_{\varphi}, \omega_{\varphi}>0$ such that

$$
\left\|\lambda R_{1}(\lambda)\right\|,\left\|\lambda^{-1} A_{1} R_{1}(\lambda)\right\| \leq M_{\varphi}|\lambda|^{-1}, \quad \lambda \in \omega_{\varphi}+\Sigma_{\frac{\pi}{2}+\varphi}
$$

Remark 2.2. In concrete problems, it happens quite often that $A_{1}$ and $B_{1}$ are bounded operators on $X$. In this situation, $\left(\mathrm{H}_{3}\right)$ holds automatically.

Prior to stating Theorem 2.3 below concerning (among others) the parabolicity of $(\mathbf{A}+\widetilde{\mathbf{A}}, \mathbf{B}+\widetilde{\mathbf{B}})$, we recall (cf., e.g., [7, p. 169]):

A linear operator $\mathbb{B}$ in a Banach space $Y$ is called $\mathbb{A}$-bounded, for a linear operator $\mathbb{A}$ in $Y$, if $\mathcal{D}(\mathbb{A}) \subset \mathcal{D}(\mathbb{B})$ and there exist constants $a, b>0$ such that

$$
\|\mathbb{B} y\| \leq a\|\mathbb{A} y\|+b\|y\|
$$

for all $y \in \mathcal{D}(\mathbb{A})$; the $\mathbb{A}$-bound of $\mathbb{B}$ is

$$
\inf \{a>0 \text {; there is } b>0 \text { such that (2.4) holds }\} \text {. }
$$


Theorem 2.3. Let $\theta \in\left(0, \frac{\pi}{2}\right]$. Suppose that $\left(\mathrm{H}_{1}\right)-\left(\mathrm{H}_{3}\right)$ hold. Let

$$
\begin{aligned}
\widetilde{A} \in \mathcal{L}\left([\mathcal{D}(A)]_{P}, E\right), & \widetilde{B} \in \mathcal{L}\left([\mathcal{D}(B)]_{P_{1}}, E\right), \\
G_{0} \in \mathcal{L}\left([\mathcal{D}(A)]_{P}, X\right), & G_{1} \in \mathcal{L}\left([\mathcal{D}(B)]_{P_{1}}, X\right), \\
\widetilde{A}_{1} \in \mathcal{L}\left(\left[\mathcal{D}\left(A_{1}\right)\right], X\right), & \widetilde{B}_{1} \in \mathcal{L}\left(\left[\mathcal{D}\left(B_{1}\right)\right], X\right),
\end{aligned}
$$

such that $\widetilde{A}, G_{0}$ are $A_{0}$-bounded with $A_{0}$-bound zero, $\widetilde{B}, G_{1}$ are $B_{0}$-bounded with $B_{0}$-bound zero, $\widetilde{A}_{1}$ is $A_{1}$-bounded with $A_{1}$-bound zero, and $\widetilde{B}_{1}$ is $B_{1}$-bounded with $B_{1}$-bound zero. Then

(1) $\mathbf{A}$ and $\mathbf{B}$ are closed, and

$$
\widetilde{\mathbf{A}} \in \mathcal{L}([\mathcal{D}(\mathbf{A})], \mathbf{E}), \quad \widetilde{\mathbf{B}} \in \mathcal{L}([\mathcal{D}(\mathbf{B})], \mathbf{E}) .
$$

(2) There exist $M_{\varphi}^{\prime}>M_{\varphi}, \omega_{\varphi}^{\prime}>\omega_{\varphi}$ such that

$$
\|\lambda \widetilde{\mathbf{R}}(\lambda)\|, \quad\left\|\lambda^{-1} \mathbf{A} \widetilde{\mathbf{R}}(\lambda)\right\|, \quad\|\mathbf{B} \widetilde{\mathbf{R}}(\lambda)\| \leq M_{\varphi}^{\prime}|\lambda|^{-1}, \quad \lambda \in \omega_{\varphi}^{\prime}+\Sigma_{\frac{\pi}{2}+\varphi} .
$$

Proof. We let

$$
\left(\begin{array}{l}
u_{n} \\
x_{n}
\end{array}\right)_{n \in N} \subset \mathcal{D}(\mathbf{B}), \lim _{n \rightarrow \infty}\left(\begin{array}{l}
u_{n} \\
x_{n}
\end{array}\right)=\left(\begin{array}{l}
u \\
x
\end{array}\right), \quad \lim _{n \rightarrow \infty} \mathbf{B}\left(\begin{array}{l}
u_{n} \\
x_{n}
\end{array}\right)=\left(\begin{array}{l}
v \\
y
\end{array}\right) .
$$

Then

$$
\left\{\begin{array} { l } 
{ \operatorname { l i m } _ { n \rightarrow \infty } x _ { n } = x , } \\
{ \operatorname { l i m } _ { n \rightarrow \infty } B _ { 1 } x _ { n } = y , }
\end{array} \quad \left\{\begin{array}{c}
\lim _{n \rightarrow \infty} u_{n}=u, \\
\lim _{n \rightarrow \infty} B u_{n}=v
\end{array}\right.\right.
$$

and $\left\{P_{1} u_{n}\right\}_{n \in N}$ is a Cauchy sequence in $X / X_{0}$ since

$$
x_{n} \in P_{1} u_{n} \quad \text { and } \quad\left\|P_{1}\left(u_{n}-u_{m}\right)\right\| \leq\left\|x_{n}-x_{m}\right\|, \quad m, n \in N .
$$

This combined with the closedness of $B_{1}$ and the completeness of $[\mathcal{D}(B)]_{P_{1}}$ indicates that

$$
x \in \mathcal{D}\left(B_{1}\right), \quad u \in \mathcal{D}(B), \quad B_{1} x=y, \quad B u=v, \quad \lim _{n \rightarrow \infty} P_{1} u_{n}=P_{1} u .
$$

We observe that

$$
\operatorname{dist}\left(x_{n}, P_{1} u\right)=\left\|P_{1} u_{n}-P_{1} u\right\|_{X / X_{1}},
$$

because of $x_{n} \in P_{1} u_{n}$. It follows that

$$
\operatorname{dist}\left(x, P_{1} u\right)=\lim _{n \rightarrow \infty} \operatorname{dist}\left(x_{n}, P_{1} u\right)=0,
$$

and therefore $x \in P_{1} u$. Thus we know that $\mathbf{B}$ is closed. A similar and simpler argument shows the closedness of $\mathbf{A}$.

Next, we observe that

$$
\begin{aligned}
& \left\|\left(\begin{array}{l}
u \\
x
\end{array}\right)\right\|_{[\mathcal{D}(\mathbf{A})]}=\|u\|+\|x\|+\|A u\|+\left\|A_{1} x\right\| \quad \text { for } \quad\left(\begin{array}{l}
u \\
x
\end{array}\right) \in \mathcal{D}(\mathbf{A}), \\
& \left\|\left(\begin{array}{l}
u \\
x
\end{array}\right)\right\|_{[\mathcal{D}(\mathbf{B})]}=\|u\|+\|x\|+\|B u\|+\left\|B_{1} x\right\| \quad \text { for } \quad\left(\begin{array}{l}
u \\
x
\end{array}\right) \in \mathcal{D}(\mathbf{B}),
\end{aligned}
$$

and that $\left(\begin{array}{l}u \\ x\end{array}\right) \in \mathcal{D}(\mathbf{A})$ (resp. $\left.\left(\begin{array}{l}u \\ x\end{array}\right) \in \mathcal{D}(\mathbf{B})\right)$ implies $x=P u$ (resp. $x \in P_{1} u$ ). From this and (2.5) - (2.7), we see easily that (2.8) is true. 
Now, fix $\varphi \in(0, \theta)$ and let $\lambda \in \omega_{\varphi}+\Sigma_{\frac{\pi}{2}+\varphi}$. If $\left(\begin{array}{l}u \\ x\end{array}\right) \in \mathcal{D}(\mathbf{A}) \cap \mathcal{D}(\mathbf{B})$, then by Lemma 2.1 (2),

$$
\begin{aligned}
& \left(\lambda^{2}+A+\lambda B\right) D_{\lambda} x=0, \\
& u-D_{\lambda} x \in(\operatorname{ker} P) \cap \mathcal{D}(A) \cap \mathcal{D}(B)=\mathcal{D}\left(A_{0}\right) \cap \mathcal{D}\left(B_{0}\right) ;
\end{aligned}
$$

so

$$
\begin{aligned}
\left(\lambda^{2}+\mathbf{A}+\lambda \mathbf{B}\right)\left(\begin{array}{l}
u \\
x
\end{array}\right) & =\left(\begin{array}{cc}
\lambda^{2}+A+\lambda B & 0 \\
0 & \left.\lambda^{2}+A_{1}+\lambda B_{1}\right)
\end{array}\right)\left(\begin{array}{l}
u \\
x
\end{array}\right) \\
& =\left(\begin{array}{cc}
\lambda^{2}+A+\lambda B & 0 \\
0 & \lambda^{2}+A_{1}+\lambda B_{1}
\end{array}\right)\left(\begin{array}{c}
u-D_{\lambda} x \\
x
\end{array}\right) \\
& =\left(\begin{array}{cc}
\lambda^{2}+A_{0}+\lambda B_{0} & 0 \\
0 & \lambda^{2}+A_{1}+\lambda B_{1}
\end{array}\right)\left(\begin{array}{c}
u-D_{\lambda} x \\
x
\end{array}\right) \\
& =\left(\begin{array}{cc}
\lambda^{2}+A_{0}+\lambda B_{0} & 0 \\
0 & \lambda^{2}+A_{1}+\lambda B_{1}
\end{array}\right)\left(\begin{array}{cc}
I & -D_{\lambda} \\
0 & I
\end{array}\right)\left(\begin{array}{l}
u \\
x
\end{array}\right) .
\end{aligned}
$$

We then get

$$
\lambda^{2}+\mathbf{A}+\lambda \mathbf{B}=\left(\begin{array}{cc}
\lambda^{2}+A_{0}+\lambda B_{0} & 0 \\
0 & \lambda^{2}+A_{1}+\lambda B_{1}
\end{array}\right)\left(\begin{array}{cc}
I & -D_{\lambda} \\
0 & I
\end{array}\right),
$$

noting that

$$
\left(\begin{array}{cc}
I & -D_{\lambda} \\
0 & I
\end{array}\right)\left(\begin{array}{l}
u \\
x
\end{array}\right) \in\left(\mathcal{D}\left(A_{0}\right) \cap \mathcal{D}\left(B_{0}\right)\right) \times\left(\mathcal{D}\left(A_{1}\right) \cap \mathcal{D}\left(B_{1}\right)\right)
$$

implies $\left(\begin{array}{l}u \\ x\end{array}\right) \in \mathcal{D}(\mathbf{A}) \cap \mathcal{D}(\mathbf{B})$. It follows that $\lambda^{2}+\mathbf{A}+\lambda \mathbf{B}$ is invertible and

$$
\begin{aligned}
\mathbf{R}(\lambda) & :=\left(\lambda^{2}+\mathbf{A}+\lambda \mathbf{B}\right)^{-1} \\
& =\left(\begin{array}{cc}
I & D_{\lambda} \\
0 & I
\end{array}\right)\left(\begin{array}{cc}
R_{0}(\lambda) & 0 \\
0 & R_{1}(\lambda)
\end{array}\right) \\
& =\left(\begin{array}{cc}
R_{0}(\lambda) & D_{\lambda} R_{1}(\lambda) \\
0 & R_{1}(\lambda)
\end{array}\right), \\
\mathbf{A R}(\lambda) & =\left(\begin{array}{cc}
A_{0} R_{0}(\lambda) & A D_{\lambda} R_{1}(\lambda) \\
0 & A_{1} R_{1}(\lambda)
\end{array}\right) .
\end{aligned}
$$

Take $\mu \in \omega_{\varphi}+\Sigma_{\frac{\pi}{2}+\varphi}$. Then

$$
A D_{\mu}, \quad B D_{\mu} \in \mathcal{L}(X, E),
$$

by Lemma 2.1 (2). Using (2.12) and $\left(\mathrm{H}_{2}\right)$, we get from (2.1),

$$
\sup \left\{\left\|D_{\lambda}\right\|+\left\|\lambda^{-2} A D_{\lambda}\right\| ; \lambda \in \omega_{\varphi}+\Sigma_{\frac{\pi}{2}+\varphi}\right\}<\infty .
$$

This combined with $\left(\mathrm{H}_{2}\right)$ and $\left(\mathrm{H}_{3}\right)$ yields that

$$
\|\lambda \mathbf{R}(\lambda)\|,\left\|\lambda^{-1} \mathbf{A R}(\lambda)\right\| \leq M^{\prime}|\lambda|^{-1}, \quad \lambda \in \omega_{\varphi}+\Sigma_{\frac{\pi}{2}+\varphi},
$$


for some constant $M^{\prime}>M_{\varphi}$. From (2.10) we have

$$
\begin{aligned}
& \widetilde{\mathbf{A}} \mathbf{R}(\lambda)=\left(\begin{array}{cc}
\widetilde{A} R_{0}(\lambda) & \widetilde{A} D_{\lambda} R_{1}(\lambda) \\
-G_{0} R_{0}(\lambda) & -G_{0} D_{\lambda} R_{1}(\lambda)+\widetilde{A}_{1} R_{1}(\lambda)
\end{array}\right), \\
& \widetilde{\mathbf{B} R}(\lambda)=\left(\begin{array}{cc}
\widetilde{B} R_{0}(\lambda) & \widetilde{B} D_{\lambda} R_{1}(\lambda) \\
-G_{1} R_{0}(\lambda) & -G_{1} D_{\lambda} R_{1}(\lambda)+\widetilde{B}_{1} R_{1}(\lambda)
\end{array}\right) .
\end{aligned}
$$

Since $\widetilde{A}$ (resp. $\widetilde{B}$ ) has $A_{0}$-bound (resp. $B_{0}$-bound) zero, there exists $a(\delta)>0$, for each $\delta>0$, such that for $\lambda \in \omega_{\varphi}+\Sigma_{\frac{\pi}{2}+\varphi}$,

$$
\begin{aligned}
\left\|\widetilde{A} R_{0}(\lambda)\right\| \leq & \delta\left\|A_{0} R_{0}(\lambda)\right\|+a(\delta)\left\|R_{0}(\lambda)\right\| \\
\leq & \delta \sup \left\{\left\|A_{0} R_{0}(\lambda)\right\| ; \quad \lambda \in \omega_{\varphi}+\Sigma_{\frac{\pi}{2}+\varphi}\right\} \\
\quad & +a(\delta) \sup \left\{\left\|\lambda^{2} R_{0}(\lambda)\right\| ; \quad \lambda \in \omega_{\varphi}+\Sigma_{\frac{\pi}{2}+\varphi}\right\}|\lambda|^{-2} \\
\left\|\lambda \widetilde{B} R_{0}(\lambda)\right\| \leq & \delta\left\|\lambda B_{0} R_{0}(\lambda)\right\|+a(\delta)\left\|\lambda R_{0}(\lambda)\right\| \\
\leq & \delta \sup \left\{\left\|\lambda B_{0} R_{0}(\lambda)\right\| ; \quad \lambda \in \omega_{\varphi}+\Sigma_{\frac{\pi}{2}+\varphi}\right\} \\
& \quad+a(\delta) \sup \left\{\left\|\lambda^{2} R_{0}(\lambda)\right\| ; \quad \lambda \in \omega_{\varphi}+\Sigma_{\frac{\pi}{2}+\varphi}\right\}|\lambda|^{-1} .
\end{aligned}
$$

Recalling $\left(\mathrm{H}_{2}\right)$, which implies

$$
\left\|B_{0} R_{0}(\lambda)\right\| \leq\left(1+2 M_{\varphi}\right)|\lambda|^{-1}, \quad \lambda \in \omega_{\varphi}+\Sigma_{\frac{\pi}{2}+\varphi},
$$

we see that the above supremums are all finite. Hence, for each $\varepsilon>0$, there exists $\beta(\varepsilon)>0$ such that for $\lambda \in \omega_{\varphi}+\Sigma_{\frac{\pi}{2}+\varphi}$,

$$
\left\|\widetilde{A} R_{0}(\lambda)\right\|, \quad\left\|\lambda \widetilde{B} R_{0}(\lambda)\right\| \leq \varepsilon+\beta(\varepsilon)|\lambda|^{-1} .
$$

The same is true of each of $\left\|G_{0} R_{0}(\lambda)\right\|,\left\|\lambda G_{1} R_{0}(\lambda)\right\|,\left\|\widetilde{A}_{1} R_{1}(\lambda)\right\|,\left\|\lambda \widetilde{B}_{1} R_{1}(\lambda)\right\|$.

Note that

$$
\widetilde{A} D_{\mu}, \quad \widetilde{B} D_{\mu} \in \mathcal{L}(X, E),
$$

by (2.5) and Lemma 2.1 (2). We deduce from (2.1), (2.12) and (2.16) that for $\lambda \in \omega_{\varphi}+\Sigma_{\frac{\pi}{2}+\varphi}$

$$
\begin{array}{r}
\left\|\widetilde{A} D_{\lambda} R_{1}(\lambda)\right\| \leq\left\|\widetilde{A} D_{\mu}\right\|\left\|R_{1}(\lambda)\right\|+\left\|\widetilde{A} R_{0}(\lambda)\right\|\left\|D_{\mu}\right\|\left\|\left(\mu^{2}-\lambda^{2}\right) R_{1}(\lambda)\right\| \\
+\left\|\widetilde{A} R_{0}(\lambda)\right\|\left\|B D_{\mu}\right\|\left\|(\mu-\lambda) R_{1}(\lambda)\right\|, \\
\left\|\lambda \widetilde{B} D_{\lambda} R_{1}(\lambda)\right\| \leq\left\|\widetilde{B} D_{\mu}\right\|\left\|\lambda R_{1}(\lambda)\right\|+\left\|\lambda \widetilde{B} R_{0}(\lambda)\right\|\left\|D_{\mu}\right\|\left\|\left(\mu^{2}-\lambda^{2}\right) R_{1}(\lambda)\right\| \\
+\left\|\lambda \widetilde{B} R_{0}(\lambda)\right\|\left\|B D_{\mu}\right\|\left\|(\mu-\lambda) R_{1}(\lambda)\right\| .
\end{array}
$$

Then, by $\left(\mathrm{H}_{3}\right)$ there is a constant $C_{0}>0$ such that for $\lambda \in \omega_{\varphi}+\Sigma_{\frac{\pi}{2}+\varphi}$,

$$
\begin{aligned}
& \left\|\widetilde{A} D_{\lambda} R_{1}(\lambda)\right\| \leq C_{0}\left(|\lambda|^{-2}+\left\|\widetilde{A} R_{0}(\lambda)\right\|\right), \\
& \left\|\lambda \widetilde{B} D_{\lambda} R_{1}(\lambda)\right\| \leq C_{0}\left(|\lambda|^{-1}+\left\|\lambda \widetilde{B} R_{0}(\lambda)\right\|\right) .
\end{aligned}
$$


Similarly, we have

$$
\begin{aligned}
& \left\|G_{0} D_{\lambda} R_{1}(\lambda)\right\| \leq C_{1}\left(|\lambda|^{-2}+\left\|G_{0} R_{0}(\lambda)\right\|\right), \quad \lambda \in \omega_{\varphi}+\Sigma_{\frac{\pi}{2}+\varphi}, \\
& \left\|\lambda G_{1} D_{\lambda} R_{1}(\lambda)\right\| \leq C_{1}\left(|\lambda|^{-1}+\left\|\lambda G_{1} R_{0}(\lambda)\right\|\right) \quad \lambda \in \omega_{\varphi}+\Sigma_{\frac{\pi}{2}+\varphi},
\end{aligned}
$$

for some constant $C_{1}>0$.

The above arguments imply the existence of a constant $\omega_{\varphi}^{\prime}>\omega_{\varphi}$ such that

$$
\|\widetilde{\mathbf{A}} \mathbf{R}(\lambda)\|+\|\lambda \widetilde{\mathbf{B}} \mathbf{R}(\lambda)\| \leq \frac{1}{2}, \quad \lambda \in \omega_{\varphi}^{\prime}+\Sigma_{\frac{\pi}{2}+\varphi},
$$

by the use of (2.14) and (2.15). Accordingly, we see that for $\lambda \in \omega_{\varphi}^{\prime}+\Sigma_{\frac{\pi}{2}+\varphi}$,

$$
\lambda^{2}+(\mathbf{A}+\widetilde{\mathbf{A}})+\lambda(\mathbf{B}+\widetilde{\mathbf{B}})=[I+\widetilde{\mathbf{A}} \mathbf{R}(\lambda)+\lambda \widetilde{\mathbf{B R}}(\lambda)]\left(\lambda^{2}+\mathbf{A}+\lambda \mathbf{B}\right)
$$

is invertible, and

$$
\widetilde{\mathbf{R}}(\lambda)=\mathbf{R}(\lambda)[I+\widetilde{\mathbf{A}} \mathbf{R}(\lambda)+\lambda \widetilde{\mathbf{B}} \mathbf{R}(\lambda)]^{-1} .
$$

This, together with (2.13), yields that for $\lambda \in \omega_{\varphi}^{\prime}+\sum_{\frac{\pi}{2}+\varphi}$,

$$
\begin{aligned}
& \|\lambda \widetilde{\mathbf{R}}(\lambda)\|,\left\|\lambda^{-1} \widetilde{\mathbf{A}} \widetilde{\mathbf{R}}(\lambda)\right\| \leq 2 M^{\prime}|\lambda|^{-1}, \\
& \|\widetilde{\mathbf{B}} \widetilde{\mathbf{R}}(\lambda)\| \leq\left\|\lambda^{-1}-\lambda \widetilde{\mathbf{R}}(\lambda)-\lambda^{-1} \widetilde{\mathbf{A}} \widetilde{\mathbf{R}}(\lambda)\right\| \leq\left(1+2 M^{\prime}\right)|\lambda|^{-1} .
\end{aligned}
$$

The proof is now complete.

By virtue of Theorem 2.3, we can obtain a fundamental solution operator of (1.8) as below.

Theorem 2.4. Assume that the conditions of Theorem 2.3 hold. Define

$$
\widetilde{\mathbf{S}}(0)=0, \quad \widetilde{\mathbf{S}}(t)=\frac{1}{2 \pi i} \int_{\Gamma} e^{\lambda t} \widetilde{\mathbf{R}}(\lambda) d \lambda \quad(t>0),
$$

where $\Gamma$ is any piecewise smooth curve in $\omega_{\varphi}+\Sigma_{\frac{\pi}{2}+\varphi}(\varphi \in(0, \theta))$ going from $\omega_{\varphi}+\infty e^{-i \delta}$ to $\omega_{\varphi}+\infty e^{i \delta}$ (for some $\delta \in\left(\frac{\pi}{2}, \frac{\pi}{2}+\varphi\right)$ ), and leaving $\omega_{\varphi}$ on its left. Then

(1) The operator function $\widetilde{\mathbf{S}}(\cdot)$ can be extended analytically to $\Sigma_{\theta}$ such that

$$
\widetilde{\mathbf{S}}(z) y \in \mathcal{D}(\mathbf{A}) \cap \mathcal{D}(\mathbf{B}) \quad \text { for } y \in Y, z \in \Sigma_{\theta},
$$

and $\mathbf{A} \widetilde{\mathbf{S}}(\cdot), \mathbf{B} \widetilde{\mathbf{S}}(\cdot)$ are analytic in $\Sigma_{\theta}$;

(2) for any $\varphi \in(0, \theta), \widetilde{\mathbf{S}}(\cdot)$ is strongly continuous in $\bar{\Sigma}_{\varphi}$;

(3) for each $y \in \overline{\mathcal{D}(\mathbf{A}) \cap \mathcal{D}(\mathbf{B})}$,

$$
\lim _{t \rightarrow 0^{+}} \widetilde{\mathbf{S}}^{\prime}(t) y=y, \quad \lim _{t \rightarrow 0^{+}} \mathbf{B} \widetilde{\mathbf{S}}(t) y=0, \quad \lim _{t \rightarrow 0^{+}} \mathbf{A} \int_{0}^{t} \widetilde{\mathbf{S}}(s) y d s=0 ;
$$

(4) for each $\varphi \in(0, \theta)$, there exists $M_{\varphi}^{\prime}>0$ such that

$$
\left\|\widetilde{\mathbf{S}}^{\prime}(z)\right\|,\|\mathbf{B} \widetilde{\mathbf{S}}(z)\|,\left\|\mathbf{A} \int_{0}^{z} \widetilde{\mathbf{S}}(\tau) d \tau\right\| \leq M_{\varphi}^{\prime} e^{\omega_{\varphi} \operatorname{Re} z}, \quad \text { for } z \in \Sigma_{\varphi} ;
$$


(5) for any $k \in\{0,1,2,3,4\}$, there exist $M, \omega>0$ such that

$$
\left\|\widetilde{\mathbf{S}}^{(k)}(t)\right\|,\left\|\mathbf{B} \widetilde{\mathbf{S}}^{(k-1)}(t)\right\|,\left\|\mathbf{A} \widetilde{\mathbf{S}}^{(k-2)}(t)\right\| \leq M t^{-(k-1)} e^{\omega t}, \quad t>0,
$$

where

$$
\widetilde{\mathbf{S}}^{(-i)}(t):=\int_{0}^{t}(t-s)^{i-1} \widetilde{\mathbf{S}}(s) d s, \quad i=1,2 ;
$$

(6) for every $z \in \Sigma_{\theta}$,

$$
\begin{gathered}
\widetilde{\mathbf{S}}^{\prime \prime}(z)+(\mathbf{B}+\widetilde{\mathbf{B}}) \widetilde{\mathbf{S}}^{\prime}(z)+(\mathbf{A}+\widetilde{\mathbf{A}}) \widetilde{\mathbf{S}}(z)=0, \\
\widetilde{\mathbf{S}}^{\prime \prime}(z) y+\widetilde{\mathbf{S}}^{\prime}(z)(\mathbf{B}+\widetilde{\mathbf{B}}) y+\widetilde{\mathbf{S}}(z)(\mathbf{A}+\widetilde{\mathbf{A}}) y=0, \quad y \in \mathcal{D}(\mathbf{A}) \cap \mathcal{D}(\mathbf{B}) .
\end{gathered}
$$

Proof. By means of Theorem [2.3, the arguments similar to those in the proof of the implication (ii) $\Longrightarrow$ (i) of [24, Theorem 1.1, Section 4.1] justify assertions (1) (4) and (6). In order to show assertion (5), we choose $\Gamma=\omega_{\varphi}+\Gamma_{1}$ with

$$
\Gamma_{1}:=\left\{\rho e^{ \pm i \frac{\pi+\varphi}{2}} ; \quad \rho \geq 1\right\} \cup\left\{e^{i \theta} ;|\theta| \leq \delta\right\} .
$$

From (2.17) we get

$$
\begin{aligned}
\widetilde{\mathbf{S}}^{(k)}(t) & =\frac{1}{2 \pi i} \int_{\Gamma} \lambda^{k} e^{\lambda t} \widetilde{\mathbf{R}}(\lambda) d \lambda \\
& =\frac{t^{-1} e^{\omega_{\varphi} t}}{2 \pi i} \int_{t \Gamma_{1}}\left(t^{-1} \mu+\omega_{\varphi}\right)^{k} e^{\mu} \widetilde{\mathbf{R}}\left(t^{-1} \mu+\omega_{\varphi}\right) d \mu \\
& =\frac{t^{-1} e^{\omega_{\varphi} t}}{2 \pi i} \int_{\Gamma_{1}}\left(t^{-1} \mu+\omega_{\varphi}\right)^{k} e^{\mu} \widetilde{\mathbf{R}}\left(t^{-1} \mu+\omega_{\varphi}\right) d \mu, \\
\mathbf{B}^{(k-1)}(t) & =\frac{1}{2 \pi i} \int_{\Gamma} \lambda^{k-1} e^{\lambda t} \mathbf{B} \widetilde{\mathbf{R}}(\lambda) d \lambda \\
& =\frac{t^{-1} e^{\omega_{\varphi} t}}{2 \pi i} \int_{\Gamma_{1}}\left(t^{-1} \mu+\omega_{\varphi}\right)^{k-1} e^{\mu} \mathbf{B} \widetilde{\mathbf{R}}\left(t^{-1} \mu+\omega_{\varphi}\right) d \mu, \\
\mathbf{A} \widetilde{\mathbf{S}}^{(k-2)}(t) & =\frac{1}{2 \pi i} \int_{\Gamma} \lambda^{k-2} e^{\lambda t} \mathbf{A} \widetilde{\mathbf{R}}(\lambda) d \lambda \\
& =\frac{t^{-1} e^{\omega_{\varphi} t}}{2 \pi i} \int_{\Gamma_{1}}\left(t^{-1} \mu+\omega_{\varphi}\right)^{k-2} e^{\mu} \mathbf{A} \widetilde{\mathbf{R}}\left(t^{-1} \mu+\omega_{\varphi}\right) d \mu .
\end{aligned}
$$

Therefore, using Theorem 2.3 yields that for $t>0$,

$$
\begin{aligned}
& \left\|\widetilde{\mathbf{S}}^{(k)}(t)\right\|,\left\|\mathbf{B} \widetilde{\mathbf{S}}^{(k-1)}(t)\right\|,\left\|\mathbf{A} \widetilde{\mathbf{S}}^{(k-2)}(t)\right\| \\
\leq & \frac{1}{2 \pi} t^{-(k-1)} e^{\omega_{\varphi} t} \int_{\Gamma}\left|\mu+t^{2}\right|^{k-2} e^{\operatorname{Re} \mu}|d \mu| \\
\leq & \operatorname{const} t^{-(k-1)}\left(1+t^{2}\right) e^{\omega_{\varphi} t} .
\end{aligned}
$$

The proof is then complete. 


\section{The MAIN THEOREM FOR PROBLEM (1.8)}

Definition 3.1. Assume that $\mathbf{A}, \mathbf{B}$ are closed, and $\widetilde{\mathbf{A}}, \widetilde{\mathbf{B}}$ satisfy (2.8). Let $h \in$ $C([0, T] ; \mathbf{E})$.

(i) A function $y(\cdot)$ is called a classical solution of (1.8) if $y(\cdot) \in C^{2}((0, T]$; $\mathbf{E}) \cap$ $C^{1}([0, T] ; \mathbf{E})$,

$$
\begin{gathered}
y(\cdot) \in C((0, T] ;[\mathcal{D}(\mathbf{A})]), \quad \int_{0} y(\sigma) d \sigma \in C([0, T] ;[\mathcal{D}(\mathbf{A})]), \\
y^{\prime}(\cdot) \in C((0, T] ;[\mathcal{D}(\mathbf{B})]), \quad y(\cdot)-y(0) \in C([0, T] ;[\mathcal{D}(\mathbf{B})]),
\end{gathered}
$$

and (1.8) is satisfied.

(ii) A function $y(\cdot)$ is called a strict solution of (1.8) if $y(\cdot) \in C^{2}([0, T] ; \mathbf{E}) \cap$ $C([0, T] ;[\mathcal{D}(\mathbf{A})]), y^{\prime}(\cdot) \in C([0, T] ;[\mathcal{D}(\mathbf{B})])$, and (1.8) is satisfied.

Remark 3.2. It can be seen from (2.8) that

(1) if $y(\cdot)$ is a classical solution of (1.8), then

$$
\begin{gathered}
\widetilde{\mathbf{B}} y^{\prime}(\cdot), \quad \widetilde{\mathbf{A}} y(\cdot) \in C((0, T] ; \mathbf{E}), \\
\widetilde{\mathbf{B}}(y(\cdot)-y(0)), \quad \widetilde{\mathbf{A}} \int_{0}^{\cdot} y(\sigma) d \sigma \in C([0, T] ; \mathbf{E}) ;
\end{gathered}
$$

(2) if $y(\cdot)$ is a strict solution of (1.8), then

$$
\widetilde{\mathbf{B}} y^{\prime}(\cdot), \quad \widetilde{\mathbf{A}} y(\cdot) \in C([0, T] ; \mathbf{E}) .
$$

Now we introduce a subset $\mathbf{\Upsilon}$ of $\mathbf{E}$, which is closely related to the Brezis-Fraenkel condition in [3] (see also [14 Appendix], [19]). Put

$$
\Upsilon:=\left\{y \in \mathcal{D}(\mathbf{B}) ; \quad \lim _{t \rightarrow 0^{+}} \Psi(t, y)=0\right\}
$$

where

$$
\begin{gathered}
\Psi(t, y):=\inf _{v \in \mathcal{D}(\mathbf{A}) \cap \mathcal{D}(\mathbf{B})}\left(t\|v\|_{[\mathcal{D}(\mathbf{A}) \cap \mathcal{D}(\mathbf{B})]}+\|y-v\|_{[\mathcal{D}(\mathbf{B})]}+t^{-1}\|y-v\|\right), \\
t \in(0, T], y \in \mathcal{D}(\mathbf{B}) .
\end{gathered}
$$

It is not difficult to see that

$$
\mathcal{D}(\mathbf{A}) \cap \mathcal{D}(\mathbf{B}) \subset \mathbf{\Upsilon} \subset \overline{\mathcal{D}(\mathbf{A}) \cap \mathcal{D}(\mathbf{B})} .
$$

We are now in a position to present our main theorem.

Theorem 3.3. Let the hypotheses of Theorem 2.3 hold, $h \in C^{\alpha}([0, T] ; \mathbf{E})(\alpha \in$

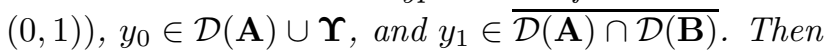

(1) problem (1.8) has a unique classical solution $y(\cdot)$, given by

$$
y(t)=\widetilde{\mathbf{C}}(t) y_{0}+\widetilde{\mathbf{S}}(t) y_{1}+\int_{0}^{t} \widetilde{\mathbf{S}}(t-s) h(s) d s, \quad t \in[0, T],
$$

where for $t \in[0, T]$,

$$
\widetilde{\mathbf{C}}(t) y_{0}:=\left\{\begin{array}{lll}
y_{0}-\int_{0}^{t} \widetilde{\mathbf{S}}(s)(\mathbf{A}+\widetilde{\mathbf{A}}) y_{0} d s, & \text { if } & y_{0} \in \mathcal{D}(\mathbf{A}), \\
\left(\widetilde{\mathbf{S}}^{\prime}(t)+\widetilde{\mathbf{S}}(t)(\mathbf{B}+\widetilde{\mathbf{B}})\right) y_{0} & \text { if } & y_{0} \in \mathbf{\Upsilon}
\end{array}\right.
$$


(2) the function $y(\cdot)$ satisfies the following regularity property and estimates:

$$
\begin{gathered}
y^{\prime \prime}(\cdot), \mathbf{B} y^{\prime}(\cdot), \mathbf{A} y(\cdot) \in C^{\alpha}([\varepsilon, T] ; \mathbf{E}), \quad \varepsilon \in(0, T) \\
\|y(t)\| \leq \operatorname{const}\left(\|h\|_{C([0, T] ; \mathbf{E})}+\left\|y_{0}\right\|_{[\mathcal{D}(\mathbf{B})]}+\left\|y_{1}\right\|\right) \\
\text { if } y_{0} \in \mathbf{\Upsilon}, t \in[0, T] ; \\
\left\|y^{\prime}(t)\right\| \leq \operatorname{const~}\left(\|h\|_{C([0, T] ; \mathbf{E})}+\left\|y_{0}\right\|_{[\mathcal{D}(\mathbf{A})]}+\left\|y_{1}\right\|\right) \\
\text { if } y_{0} \in \mathcal{D}(\mathbf{A}), t \in[0, T] \\
\leq y^{\prime \prime}(t)\|+\| y^{\prime}(t)\left\|_{[\mathcal{D}(\mathbf{B})]}+\right\| y(t) \|_{[\mathcal{D}(\mathbf{A})]} \\
\text { const }\left(\|h\|_{C^{\alpha}([0, T] ; \mathbf{E})}+\left\|y_{0}\right\|_{[\mathcal{D}(\mathbf{A})]}+\left\|y_{1}\right\|_{[\mathcal{D}(\mathbf{A}) \cap \mathcal{D}(\mathbf{B})]}\right), \\
\text { if } y_{0} \in \mathcal{D}(\mathbf{A}), y_{1} \in \mathcal{D}(\mathbf{A}) \cap \mathcal{D}(\mathbf{B}), t \in(0, T] .
\end{gathered}
$$

(3) the function $y(t)$ is a strict solution of 1.8 provided $y_{0} \in \mathcal{D}(\mathbf{A}), y_{1} \in \mathbf{\Upsilon}$, and

$$
(\mathbf{A}+\widetilde{\mathbf{A}}) y_{0}+(\mathbf{B}+\widetilde{\mathbf{B}}) y_{1}-h(0) \in \overline{\mathcal{D}(\mathbf{A}) \cap \mathcal{D}(\mathbf{B})}
$$

Proof. We will use freely the closedness of A, B and the fact (2.8) concerning $\widetilde{\mathbf{A}}$ and $\widetilde{\mathbf{B}}$. Put

$$
y_{*}(t):=\int_{0}^{t} \widetilde{\mathbf{S}}(t-s) h(s) d s, \quad t \in[0, T] .
$$

We then have (noting $\widetilde{\mathbf{S}}(0)=0$ )

$$
\begin{gathered}
y_{*}(t)=\int_{0}^{t} \widetilde{\mathbf{S}}(\sigma) h(t) d \sigma+\int_{0}^{t} \widetilde{\mathbf{S}}(t-\sigma)(h(\sigma)-h(t)) d \sigma, \quad t \in[0, T], \\
y_{*}^{\prime}(t)=\widetilde{\mathbf{S}}(t) h(t)+\int_{0}^{t} \widetilde{\mathbf{S}}^{\prime}(t-\sigma)(h(\sigma)-h(t)) d \sigma, \quad t \in[0, T], \\
y_{*}^{\prime \prime}(t)=\widetilde{\mathbf{S}}^{\prime}(t) h(t)+\int_{0}^{t} \widetilde{\mathbf{S}}^{\prime \prime}(t-\sigma)(h(\sigma)-h(t)) d \sigma, \quad t \in(0, T],
\end{gathered}
$$

in view of the estimates

$$
\|h(\sigma)-h(t)\| \leq \mathrm{const}(t-\sigma)^{\alpha}, \quad 0 \leq \sigma \leq t \leq T,
$$

and (2.20). Thus, we infer by (3.13), (2.20), (2.21) and Theorem 2.4 (1) and (2) that

$$
\begin{gathered}
y_{*}^{\prime \prime}(\cdot), \quad \mathbf{B} y_{*}^{\prime}(\cdot), \quad \mathbf{A} y_{*}(\cdot) \in C((0, T] ; \mathbf{E}), \\
y_{*}^{\prime}(\cdot), \quad \mathbf{B} y_{*}(\cdot), \quad \mathbf{A} \int_{0}^{\cdot} y_{*}(\sigma) d \sigma \in C([0, T] ; \mathbf{E}), \\
y_{*}^{\prime \prime}(t)+(\mathbf{B}+\widetilde{\mathbf{B}}) y_{*}^{\prime}(t)+(\mathbf{A}+\widetilde{\mathbf{A}}) y_{*}(t)=h(t), \quad t \in(0, T] .
\end{gathered}
$$

Clearly

$$
y_{*}(0)=0, \quad y_{*}^{\prime}(0)=0,
$$


by (3.10), (3.11) and (2.17). Next, we fix $\varepsilon \in(0, T)$. Using (3.12), (3.13) and (2.20) yields that for $\varepsilon \leq s<t \leq T$,

$$
\begin{aligned}
& \left\|y_{*}^{\prime \prime}(t)-y_{*}^{\prime \prime}(s)\right\| \\
& \leq\left\|\widetilde{\mathbf{S}}^{\prime}(t)\right\|\|h(t)-h(s)\|+\left\|\int_{s}^{t} \widetilde{\mathbf{S}}^{\prime \prime}(\sigma) d \sigma\right\|\|h(s)\| \\
& +\int_{0}^{s}\left\|\widetilde{\mathbf{S}}^{\prime \prime}(t-\sigma)-\widetilde{\mathbf{S}}^{\prime \prime}(s-\sigma)\right\|\|h(\sigma)-h(s)\| d \sigma \\
& +\left\|\int_{0}^{s} \widetilde{\mathbf{S}}^{\prime \prime}(t-\sigma) d \sigma\right\|\|h(s)-h(t)\| \\
& +\int_{s}^{t}\left\|\widetilde{\mathbf{S}}^{\prime \prime}(t-\sigma)\right\|\|h(\sigma)-h(t)\| d \sigma \\
& \leq \text { const }\left[(t-s)^{\alpha}+\int_{s}^{t} \sigma^{-1} d \sigma+\int_{0}^{s}\left|\int_{s-\sigma}^{t-\sigma} \tau^{-2} d \tau\right|(s-\sigma)^{\alpha} d \sigma\right. \\
& \left.+\|\widetilde{\mathbf{S}}(t-s)-\widetilde{\mathbf{S}}(t)\|(t-s)^{\alpha}+\int_{s}^{t}(t-\sigma)^{\alpha-1} d \sigma\right] \\
& \leq \text { const }\left[(t-s)^{\alpha}+\varepsilon^{-1}(t-s)+(t-s) \int_{0}^{s}(t-\sigma)^{-1}(s-\sigma)^{\alpha-1} d \sigma\right] \\
& \leq \text { const }(t-s)^{\alpha} \text {. }
\end{aligned}
$$

In a similar way, we obtain from (3.10) and (3.11)

$$
\left\|\mathbf{B} y_{*}^{\prime}(t)-\mathbf{B} y_{*}^{\prime}(s)\right\|,\left\|\mathbf{A} y_{*}(t)-\mathbf{A} y_{*}(s)\right\| \leq \operatorname{const}(t-s)^{\alpha}, \quad \varepsilon \leq s<t \leq T .
$$

Therefore

$$
y_{*}^{\prime \prime}(\cdot), \mathbf{B} y_{*}^{\prime}(\cdot), \mathbf{A} y_{*}(\cdot) \in C^{\alpha}([\varepsilon, T] ; \mathbf{E}), \quad \varepsilon \in(0, T) .
$$

We now take care of $\widetilde{\mathbf{C}}(\cdot) y_{0}$ and $\widetilde{\mathbf{S}}(\cdot) y_{1}$. By (3.4) and the related properties of $\widetilde{\mathbf{S}}(\cdot)$ (see Theorem 2.4), we get

$$
\begin{gathered}
\widetilde{\mathbf{C}}(0) y_{0}=y_{0}, \quad \widetilde{\mathbf{S}}(0) y_{1}=0, \quad \widetilde{\mathbf{C}}^{\prime}(0) y_{0}=0, \quad \widetilde{\mathbf{S}}^{\prime}(0) y_{1}=y_{1}, \\
\widetilde{\mathbf{C}}^{\prime \prime}(\cdot) y_{0}, \mathbf{B} \widetilde{\mathbf{C}}^{\prime}(\cdot) y_{0}, \mathbf{A} \widetilde{\mathbf{C}}(\cdot) y_{0} \in C((0, T] ; \mathbf{E}), \\
\widetilde{\mathbf{S}}^{\prime \prime}(\cdot) y_{1}, \mathbf{B} \widetilde{\mathbf{S}}^{\prime}(\cdot) y_{1}, \mathbf{A} \widetilde{\mathbf{S}}(\cdot) y_{1} \in C((0, T] ; \mathbf{E}),
\end{gathered}
$$

and

$$
\begin{aligned}
& \widetilde{\mathbf{C}}^{\prime \prime}(t) y_{0}+\widetilde{\mathbf{S}}^{\prime \prime}(t) y_{1}+(\mathbf{B}+\widetilde{\mathbf{B}})\left(\widetilde{\mathbf{C}}^{\prime}(t) y_{0}+\widetilde{\mathbf{S}}^{\prime}(t) y_{1}\right) \\
& +(\mathbf{A}+\widetilde{\mathbf{A}})\left(\widetilde{\mathbf{C}}(t) y_{0}+\widetilde{\mathbf{S}}(t) y_{1}\right) \\
=0, \quad t & \in(0, T] .
\end{aligned}
$$


Moreover, using (2.20), we see easily that for $\varepsilon \leq s<t \leq T$,

$$
\left.\begin{array}{l}
\left\|\widetilde{\mathbf{C}}^{\prime \prime}(t) y_{0}-\widetilde{\mathbf{C}}^{\prime \prime}(s) y_{0}\right\| \\
\left\|\mathbf{B} \widetilde{\mathbf{C}}^{\prime}(t) y_{0}-\mathbf{B} \widetilde{\mathbf{C}}^{\prime}(s) y_{0}\right\| \\
\left\|\mathbf{A} \widetilde{\mathbf{C}}(t) y_{0}-\mathbf{A} \widetilde{\mathbf{C}}(s) y_{0}\right\|
\end{array}\right\} \leq \text { const }(t-s),
$$

In the following, we will show that

$$
\widetilde{\mathbf{C}}^{\prime}(t) y_{0}, \mathbf{B}\left(\widetilde{\mathbf{C}}(t) y_{0}-y_{0}\right), \mathbf{A} \int_{0}^{t} \widetilde{\mathbf{C}}(\sigma) y_{0} d \sigma \longrightarrow 0
$$

as $t \rightarrow 0^{+}$. When $y_{0} \in \mathcal{D}(\mathbf{A})$, (3.25) follows immediately from (3.4) and Theorem 2.4 (2) and (4). Now let $y_{0} \in \boldsymbol{\Upsilon}$. Making use of (2.20), (2.22) and noting $\widetilde{\mathbf{S}}(0)=0, \widetilde{\mathbf{S}}^{\prime}(0) v=v$ for $v \in \mathcal{D}(\mathbf{A}) \cap \mathcal{D}(\mathbf{B})$, we obtain

$$
\begin{aligned}
& \left\|\widetilde{\mathbf{C}}^{\prime}(t) y_{0}\right\| \\
& =\inf _{v \in \mathcal{D}(\mathbf{A}) \cap \mathcal{D}(\mathbf{B})}\left\|\widetilde{\mathbf{C}}^{\prime}(t)\left(y_{0}-v\right)-\widetilde{\mathbf{S}}(t)(\mathbf{A}+\widetilde{\mathbf{A}}) v\right\| \\
& \leq \text { const } \inf _{v \in \mathcal{D}(\mathbf{A}) \cap \mathcal{D}(\mathbf{B})}\left(t^{-1}\left\|y_{0}-v\right\|+\left\|(\mathbf{B}+\widetilde{\mathbf{B}})\left(y_{0}-v\right)\right\|+t\|(\mathbf{A}+\widetilde{\mathbf{A}}) v\|\right) \\
& \leq \text { const } \Psi\left(t, y_{0}\right), \quad t \in(0, T] \quad \text { (by (3.2)), } \\
& \left\|\mathbf{B}\left(\widetilde{\mathbf{C}}(t) y_{0}-y_{0}\right)\right\| \\
& =\inf _{v \in \mathcal{D}(\mathbf{A}) \cap \mathcal{D}(\mathbf{B})}\left\|\mathbf{B} \widetilde{\mathbf{C}}(t)\left(y_{0}-v\right)-\mathbf{B} \int_{0}^{t} \widetilde{\mathbf{S}}(\sigma)(\mathbf{A}+\widetilde{\mathbf{A}}) v d \sigma+\mathbf{B}\left(v-y_{0}\right)\right\| \\
& \leq \text { const } \Psi\left(t, y_{0}\right), \quad t \in(0, T] \text {, } \\
& \left\|\mathbf{A} \int_{0}^{t} \widetilde{\mathbf{C}}(\sigma) y_{0} d \sigma\right\| \\
& =\inf _{v \in \mathcal{D}(\mathbf{A}) \cap \mathcal{D}(\mathbf{B})} \| \mathbf{A}\left(\widetilde{\mathbf{S}}(t)+\int_{0}^{t} \widetilde{\mathbf{S}}(\sigma)(\mathbf{B}+\widetilde{\mathbf{B}}) d \sigma\right)\left(y_{0}-v\right) \\
& -\mathbf{A} \int_{0}^{t}(t-\sigma) \widetilde{\mathbf{S}}(\sigma)(\mathbf{A}+\widetilde{\mathbf{A}}) v d \sigma+t \mathbf{A} v \| \\
& \leq \text { const } \Psi\left(t, y_{0}\right), \quad t \in(0, T] .
\end{aligned}
$$

This leads to (3.25) in view of the definition of $\Upsilon$ (see (3.1)). Combining (2.18), (3.14) - (3.17), (3.19) - (3.22), and (3.25) together, we deduce that the function $y(\cdot)$ defined by (3.3) is a classical solution of problem (1.8). 
In order to show the uniqueness, let $v(\cdot)$ be another classical solution of (1.8). Then

$v^{\prime}(t)-y^{\prime}(t)+(\mathbf{B}+\widetilde{\mathbf{B}})(v(t)-y(t))+(\mathbf{A}+\widetilde{\mathbf{A}}) \int_{0}^{t}(v(s)-y(s)) d s=0, \quad t \in[0, T]$.

So a calculation involving integration by parts shows that for $t \in[0, T], \lambda$ large enough,

$$
\begin{aligned}
& \left(\lambda+(\mathbf{B}+\widetilde{\mathbf{B}})+\lambda^{-1}(\mathbf{A}+\widetilde{\mathbf{A}})\right) \int_{0}^{t} e^{\lambda(t-s)}(v(s)-y(s)) d s \\
= & -v(t)+y(t)+\lambda^{-1}(\mathbf{A}+\widetilde{\mathbf{A}}) \int_{0}^{t}(v(\sigma)-y(\sigma)) d \sigma .
\end{aligned}
$$

Hence for $t \in[0, T]$,

$$
\lim _{\lambda \rightarrow \infty} e^{-\lambda} \int_{0}^{t} e^{\lambda(t-s)}(v(s)-y(s)) d s=0
$$

since $\lim _{\lambda \rightarrow \infty} \lambda^{2} e^{-\lambda} \widetilde{\mathbf{R}}(\lambda) w=0(w \in \mathbf{E})$. This yields that $v(t)=y(t)$ for all $t \in[0, T]$, in view of [20, Lemma 1.1, p. 100]. Therefore, assertion (1) is valid. The regularity property (3.5) comes from (3.18), (3.23) and (3.24). Based on the expression (3.3) of $y(t)$, we derive the estimates (3.6) - (3.8) by (2.19) and (2.21).

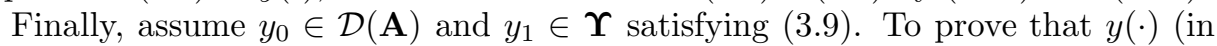
this case) is a strict solution, we observe by (3.3), (2.22) and (3.12) that

$$
\begin{aligned}
& y^{\prime \prime}(t)=--\widetilde{\mathbf{S}}^{\prime}(t) \\
&\left.+(\mathbf{A}+\widetilde{\mathbf{A}}) y_{0}+(\mathbf{B}+\widetilde{\mathbf{B}}) y_{1}-h(0)\right) \\
&+\widetilde{\mathbf{S}}^{\prime \prime}(t) y_{1}+\widetilde{\mathbf{S}}^{\prime}(t)(\mathbf{B}+\widetilde{\mathbf{B}}) y_{1}+\widetilde{\mathbf{S}}^{\prime}(t)(h(t)-h(0)) \\
&+\int_{0}^{t} \widetilde{\mathbf{S}}^{\prime \prime}(t-\sigma)(h(\sigma)-h(t)) d \sigma, \quad t \in(0, T] .
\end{aligned}
$$

The same reasoning as for (3.25) (in the case of $y_{0} \in \mathbf{\Upsilon}$ ) gives that

$$
\lim _{t \rightarrow 0^{+}}\left(\widetilde{\mathbf{S}}^{\prime \prime}(t) y_{1}+\widetilde{\mathbf{S}}^{\prime}(t)(\mathbf{B}+\widetilde{\mathbf{B}}) y_{1}\right)=0 .
$$

Therefore

$$
\lim _{t \rightarrow 0^{+}} y^{\prime \prime}(t)=-(\mathbf{A}+\widetilde{\mathbf{A}}) y_{0}-(\mathbf{B}+\widetilde{\mathbf{B}}) y_{1}+h(0),
$$

by (2.18) - 2.20) and (3.13). Analogously, we obtain

$$
\lim _{t \rightarrow 0^{+}} \mathbf{B} y^{\prime}(t)=\mathbf{B} y_{1}, \quad \lim _{t \rightarrow 0^{+}} \mathbf{A} y(t)=\mathbf{A} y_{0} .
$$

Thus, (3.26) and (3.27) together with assertion (1) justify assertion (3). This finishes the proof.

\section{EXAMPLES}

In this section, we present three examples, which do not aim at generality but indicate how our theorems can be applied to concrete problems. 
Example 4.1. We consider a linear viscoelastic beam with a tip mass whose dynamic evolution is described by the following system:

$$
\begin{cases}\partial_{t}^{2} u+\partial_{\xi}^{4} u+\beta \partial_{\xi}^{4} \partial_{t} u=f, & t \in[0, T], \xi \in(0,1), \\ u(t, 0)=\partial_{\xi} u(t, 0)=0, & t \in[0, T], \\ \kappa \partial_{t}^{2} u(t, 1)-\partial_{\xi}^{3} u(t, 1)-\beta \partial_{\xi}^{3} \partial_{t} u(t, 1)=0, & t \in[0, T], \\ \gamma \partial_{t}^{2} \partial_{\xi} u(t, 1)+\partial_{\xi}^{2} u(t, 1)+\beta \partial_{\xi}^{2} \partial_{t} u(t, 1)=0, & t \in[0, T], \\ u(0, \xi)=\varphi_{0}(\xi), \partial_{t} u(0, \xi)=\varphi_{1}(\xi), & \xi \in[0,1],\end{cases}
$$

where $\beta, \kappa$ and $\gamma$ are positive constants, and $f \in C^{\alpha}\left([0, T] ; L^{2}(0,1)\right)(\alpha \in(0,1))$.

Take

$$
\begin{aligned}
& E=L^{2}(0,1), \quad X=\mathbf{C}^{2}, \\
& A=\frac{d^{4}}{d \xi^{4}} \quad \text { with } \quad \mathcal{D}(A)=\left\{\varphi \in H^{4}(0,1) ; \quad \varphi(0)=\varphi^{\prime}(0)=0\right\} \\
& P \varphi=\left(\begin{array}{c}
\varphi(1) \\
\varphi^{\prime}(1)
\end{array}\right) \quad \text { for } \quad \varphi \in \mathcal{D}(P):=\mathcal{D}(A), \\
& G_{0} \varphi=\left(\begin{array}{c}
\kappa^{-1} \varphi^{\prime \prime \prime}(1) \\
-\gamma^{-1} \varphi^{\prime \prime}(1)
\end{array}\right) \quad \text { for } \quad \varphi \in \mathcal{D}\left(G_{0}\right):=\mathcal{D}(A), \\
& B=\beta A, \quad P_{1}=P, \quad G_{1}=\beta G_{0}, \\
& A_{1}=0, \quad B_{1}=0, \quad \widetilde{A}=0, \quad \widetilde{A}_{1}=0, \quad \widetilde{B}=0, \quad \widetilde{B}_{1}=0 .
\end{aligned}
$$

Then we see easily that $B_{0}=\beta A_{0}$ (see (1.7)),

$$
A_{0}=\frac{d^{4}}{d \xi^{4}} \quad \text { with } \quad \mathcal{D}\left(A_{0}\right)=\left\{\varphi \in H^{4}(0,1) ; \quad \varphi(0)=\varphi^{\prime}(0)=\varphi(1)=\varphi^{\prime}(1)=0\right\},
$$

and

$$
\|\cdot\|_{A, P} \sim\|\cdot\|_{B, P_{1}} \sim\|\cdot\|_{H^{4}} .
$$

Obviously $\left(\mathrm{H}_{1}\right),\left(\mathrm{H}_{3}\right)$ and (2.6) hold. From the fact that $-B_{0}$ generates a strongly continuous analytic semigroup on $L^{2}(0,1)$, it follows that $\left(\mathrm{H}_{2}\right)$ holds too (cf. [24] Corollary 1.6, p. 149]). It is clear that $G_{0}:\left[\mathcal{D}\left(A_{0}\right)\right] \rightarrow \mathbf{C}^{2}$ is bounded and so compact. By [7] Lemma 2.16, p. 179] (with a slight improvement), we deduce that $G_{0}$ is $A_{0}$-bounded with $A_{0}$-bound zero. Likewise, $G_{1}$ is $B_{0}$-bounded with $B_{0}$-bound zero. Moreover, we note that $\overline{\mathcal{D}(\mathbf{A}) \cap \mathcal{D}(\mathbf{B})}=\mathbf{E}$ (see Section 1 for the definitions of $\mathbf{A}, \mathbf{B}$ and $\mathbf{E})$, since $\mathcal{D}\left(A_{0}\right) \cap \mathcal{D}\left(B_{0}\right)$ is dense in $L^{2}(0,1)$ and $P(\mathcal{D}(A) \cap \mathcal{D}(B))=X$. Setting

$$
y(t)=\left(\begin{array}{c}
u(t) \\
x(t)
\end{array}\right), \quad u(t)=u(t, \cdot), \quad x(t)=\left(\begin{array}{c}
u(t, 1) \\
\partial_{\xi} u(t, 1)
\end{array}\right), \quad t \in[0, T],
$$

we apply Theorem 3.3 to conclude:

For every $\varphi_{0}, \varphi_{1} \in H^{4}(0,1)$ with $\varphi_{i}(0)=\varphi_{i}^{\prime}(0)=0(i=0,1)$, problem (4.1) has a unique solution

$$
u \in C^{2}\left([0, T] ; L^{2}(0,1)\right) \cap C^{1}\left([0, T] ; H^{4}(0,1)\right) ;
$$


moreover,

$$
\begin{gathered}
\partial_{t}^{2} u \in C^{\alpha}\left([\varepsilon, T] ; L^{2}(0,1)\right), \quad \partial_{t} u \in C^{\alpha}\left([\varepsilon, T] ; H^{4}(0,1)\right), \quad \varepsilon \in(0, T), \\
\left\|\partial_{t}^{2} u(t, \cdot)\right\|_{L^{2}(0,1)}+\left\|\partial_{t} u(t, \cdot)\right\|_{H^{4}(0,1)} \\
\leq \text { const }\left(\|f\|_{C^{\alpha}\left([0, T] ; L^{2}(0,1)\right)}+\left\|\varphi_{0}\right\|_{H^{4}(0,1)}+\left\|\varphi_{1}\right\|_{H^{4}(0,1)}\right), \quad t \in[0, T] .
\end{gathered}
$$

For the uniqueness, we have made the observation that if $u$ is a solution of problem (4.1) satisfying (4.2), then

$$
x^{\prime}(t)=P_{1} u^{\prime}(t) \quad(t \in[0, T]), \quad x(0)=\left(\begin{array}{l}
\varphi_{0}(1) \\
\varphi_{0}^{\prime}(1)
\end{array}\right), \quad x^{\prime}(0)=\left(\begin{array}{c}
\varphi_{1}(1) \\
\varphi_{1}^{\prime}(1)
\end{array}\right) .
$$

Remark 4.2. In [1, Theorem 5.1], problem (4.1) was discussed in a much weaker form. The weak solution $v$ obtained there satisfies that

$$
v, v^{\prime} \in L^{2}((0, T) ; \mathcal{E}), \quad(Q v)^{\prime},(Q v)^{\prime \prime} \in L^{2}\left((0, T) ; \mathcal{E}^{\prime}\right),
$$

and

$$
\left\{\begin{array}{l}
(Q v)^{\prime \prime}+(M v)^{\prime}+N v=f \quad \text { in } L^{2}\left((0, T) ; \mathcal{E}^{\prime}\right) \\
Q v(0)=Q \varphi_{0}, \quad(Q v)^{\prime}(0)=Q \varphi_{1}
\end{array}\right.
$$

where $\mathcal{E}:=\left\{\varphi \in H^{2}(0,1) ; \quad \varphi(0)=\varphi^{\prime}(0)=0\right\}$, the dual space $\mathcal{E}^{\prime} \supset L^{2}(0,1) \supset \mathcal{E}$, and the operators $Q, M, N: \mathcal{E} \rightarrow \mathcal{E}^{\prime}$ are defined by

$$
\begin{aligned}
& \langle Q \varphi, \psi\rangle=\int_{0}^{1} \varphi \psi d \xi+\kappa \phi(1) \psi(1)+\gamma \phi^{\prime}(1) \psi^{\prime}(1), \\
& \langle M \varphi, \psi\rangle=\int_{0}^{1} \beta \varphi^{\prime \prime} \psi^{\prime \prime} d \xi \\
& \langle N \varphi, \psi\rangle=\int_{0}^{1} \varphi^{\prime \prime} \psi^{\prime \prime} d \xi, \quad \varphi, \psi \in \mathcal{E} .
\end{aligned}
$$

Example 4.3. Let $\Omega$ be a bounded domain in $R^{n}$ with smooth boundary $\partial \Omega$, and let $\rho>0$. We consider the mixed boundary control problem for a structurally damped plate-like equation:

$$
\begin{cases}\partial_{t}^{2} u+\Delta^{2} u-\rho \Delta \partial_{t} u=0, & \text { in }[0, T] \times \Omega, \\ \partial_{t}^{2}\left(\left.u\right|_{\partial \Omega}\right)=w, & \text { in }[0, T] \times \partial \Omega, \\ \left.\Delta u\right|_{\partial \Omega}=0, & \text { in }[0, T] \times \partial \Omega, \\ u(0, \cdot)=\varphi_{0}, \quad \partial_{t} u(0, \cdot)=\varphi_{1}, & \text { in } \Omega,\end{cases}
$$

where $w$ is the control force.

The objective is to show that problem (4.3) (with a suitable $w$ ) is well posed in $L^{p}(\Omega)(1<p<\infty)$.

We consider the case where $w$ is built up by a feedback control law:

$$
w=\langle\Delta u, a\rangle b+g
$$

with

$$
\begin{gathered}
a \in L^{q}(\Omega)\left(\frac{1}{q}+\frac{1}{p}=1\right), \quad b \in W^{2, p}(\partial \Omega), \\
g \in C^{\alpha}\left([0, T] ; W^{2, p}(\partial \Omega)\right) \quad(\alpha \in(0,1)) .
\end{gathered}
$$


When $a=0$, 4.3. becomes an open loop problem.

In order to apply our theorems, we take

$$
\begin{aligned}
& E=L^{p}(\Omega), \quad X=W^{2-\frac{1}{p}, p}(\partial \Omega), \\
& B=-\rho \Delta \quad \text { with } \mathcal{D}(B)=W^{2, p}(\Omega), \\
& A=\Delta^{2} \quad \text { with } \mathcal{D}(A)=\left\{\varphi \in \mathcal{D}\left(B^{2}\right) ;\left.\quad \Delta \varphi\right|_{\partial \Omega}=0\right\}, \\
& G_{0} \varphi=\langle\Delta \varphi, a\rangle b \quad \text { for } \quad \varphi \in \mathcal{D}\left(G_{0}\right):=\mathcal{D}(A), \\
& P \varphi=\left.\varphi\right|_{\partial \Omega} \text { for } \varphi \in \mathcal{D}(P):=\mathcal{D}(A), \quad P_{1}=P, \\
& A_{1}=0, \quad B_{1}=0, \quad \widetilde{A}=0, \quad \widetilde{A}_{1}=0, \quad \widetilde{B}=0, \quad \widetilde{B}_{1}=0, \quad G_{1}=0 .
\end{aligned}
$$

We claim that $\left(\mathrm{H}_{1}\right)$ is satisfied. In fact, a trace theorem [23, Section 5.5.2, pp. 390, 391] says that

$$
\mathcal{P}: \varphi \longmapsto\left(\Delta \varphi,\left.\varphi\right|_{\partial \Omega}\right)
$$

is an isomorphic mapping from $W^{2, p}(\Omega)$ onto $L^{p}(\Omega) \times W^{2-\frac{1}{p}, p}(\partial \Omega)$. Hence, given $x \in W^{2-\frac{1}{p}, p}(\partial \Omega)$, there exists $\varphi \in W^{2, p}(\Omega)$ such that

$$
\Delta \varphi=0,\left.\quad \varphi\right|_{\partial \Omega}=x .
$$

It follows immediately that

$$
\varphi \in \mathcal{D}(A) \text { and } P \varphi=x .
$$

So $P(\mathcal{D}(A) \cap \mathcal{D}(B))=X$. Next we show the completeness of $[\mathcal{D}(A)]_{P}$. To this end, we take a Cauchy sequence $\left\{\psi_{n}\right\}_{n \in N}$ in $[\mathcal{D}(A)]_{P}$. Then, there exist $r, r_{0} \in L^{p}(\Omega)$ and $v \in W^{2-\frac{1}{p}, p}(\partial \Omega)$ such that

$$
\begin{gathered}
\lim _{n \rightarrow \infty}\left\|\psi_{n}-r\right\|_{L^{p}(\Omega)}=0, \\
\lim _{n \rightarrow \infty}\left\|\Delta^{2} \psi_{n}-r_{0}\right\|_{L^{p}(\Omega)}=0, \\
\lim _{n \rightarrow \infty}\left\|\left.\psi_{n}\right|_{\partial \Omega}-v\right\|_{W^{2-\frac{1}{p}, p}(\partial \Omega)}=0, \\
\left.\Delta \psi_{n}\right|_{\partial \Omega}=0 .
\end{gathered}
$$

According to (4.5) and 4.7), the isomorphism $\mathcal{P}$ implies the existence of $r_{1}, r_{2} \in$ $W^{2, p}(\Omega)$ such that

$$
\begin{gathered}
\lim _{n \rightarrow \infty}\left\|\Delta \psi_{n}-r_{1}\right\|_{L^{p}(\Omega)}=0, \\
\Delta r_{1}=r_{0},\left.\quad r_{1}\right|_{\partial \Omega}=0 .
\end{gathered}
$$

Using (4.4), (4.6) and (4.8) yields that

$$
r \in W^{2, p}(\Omega), \quad \Delta r=r_{1},\left.\quad r\right|_{\partial \Omega}=v .
$$

From this, (4.9) and (4.4) - (4.7), we deduce that

$$
r \in \mathcal{D}(A) \quad \text { and } \quad \lim _{n \rightarrow \infty}\left\|\psi_{n}-r\right\|_{A, P}=0 .
$$


Therefore $[\mathcal{D}(A)]_{P}$ is complete. The completeness of $[\mathcal{D}(B)]_{P_{1}}$ can be verified in the same way. Moreover, using the $\mathcal{P}$ again we find that

$$
\|\cdot\|_{A, P} \sim\|\cdot\|_{W^{2, p}(\Omega)}+\|\Delta \cdot\|_{W^{2, p}(\Omega)}, \quad\|\cdot\|_{B, P_{1}} \sim\|\cdot\|_{W^{2, p}(\Omega)} .
$$

Clearly $B_{0}:=\left.B\right|_{\operatorname{ker} P_{1}}=-\rho \Delta_{D}$ and $A_{0}:=\left.A\right|_{\operatorname{ker} P}=\Delta_{D}^{2}\left(\Delta_{D}\right.$ is the Dirichlet Laplacian). By [14, Theorem 3.4], $\left(\mathrm{H}_{2}\right)$ holds. The first equivalent relation in (4.10) tells us that $G_{0} \in \mathcal{L}\left([\mathcal{D}(A)]_{P}, X\right)$. Obviously, $G_{0}$ is relatively $\Delta_{D}^{2}$-bounded with $\Delta_{D}^{2}$-bound zero. Thus the hypotheses of Theorem 2.3 are fulfilled. So Theorem 2.3 is applicable to this situation, in which $\overline{\mathcal{D}(\mathbf{A}) \cap \mathcal{D}(\mathbf{B})}=\mathbf{E}$,

$$
y(t)=\left(\begin{array}{l}
u(t) \\
x(t)
\end{array}\right), \quad u(t)=u(t, \cdot), \quad x(t):=\left.u(t, \cdot)\right|_{\partial \Omega}, \quad t \in[0, T] .
$$

Noting (4.10), we then obtain the following conclusion:

For every $\varphi_{0}, \varphi_{1} \in W^{2, p}(\Omega)$ with $\Delta \varphi_{0}, \Delta \varphi_{1} \in W^{2, p}(\Omega)$ and $\left.\Delta \varphi_{0}\right|_{\partial \Omega}=\left.\Delta \varphi_{1}\right|_{\partial \Omega}$ $=0$, problem (4.3) has a unique solution

$$
u \in C^{2}\left([0, T] ; L^{p}(\Omega)\right) \cap C^{1}\left([0, T] ; W^{2, p}(\Omega)\right) ;
$$

moreover,

$$
\begin{aligned}
& \Delta u \in C\left([0, T] ; W^{2, p}(\Omega)\right), \\
& \partial_{t}^{2} u \in C^{\alpha}\left([\varepsilon, T] ; L^{p}(\Omega)\right), \quad \partial_{t} u, \Delta u \in C^{\alpha}\left([\varepsilon, T] ; W^{2, p}(\Omega)\right), \quad \varepsilon \in(0, T),
\end{aligned}
$$

and for $t \in[0, T]$

$$
\begin{aligned}
& \left\|\partial_{t}^{2} u(t, \cdot)\right\|_{L^{p}(\Omega)}+\left\|\partial_{t} u(t, \cdot)\right\|_{W^{2, p}(\Omega)}+\|\Delta u(t, \cdot)\|_{W^{2, p}(\Omega)} \\
\leq & \operatorname{const}\left(\|g\|_{C^{\alpha}\left([0, T] ; W^{2, p}(\partial \Omega)\right)}+\sum_{j=0}^{1}\left\|\varphi_{j}\right\|_{W^{2, p}(\Omega)}+\left\|\Delta \varphi_{j}\right\|_{W^{2, p}(\Omega)}\right) .
\end{aligned}
$$

Here, for getting the uniqueness we used the fact that if $u$ is a solution of problem (4.3) satisfying (4.11), then $\left.\left(\partial_{t} u(t, \cdot)\right)\right|_{\partial \Omega}=\partial_{t}\left(\left.u(t, \cdot)\right|_{\partial \Omega}\right)$, by virtue of the isomorphism $\mathcal{P}$, and therefore

$$
x^{\prime}(t)=P_{1} u^{\prime}(t) \quad(t \in[0, T]), \quad x(0)=\left.\varphi_{0}\right|_{\partial \Omega}, \quad x^{\prime}(0)=\left.\varphi_{1}\right|_{\partial \Omega} .
$$

Remark 4.4. To our knowledge, the result in Example 4.3 is new even for the case of $p=2$ and $a=0$.

Example 4.5. Let $\rho>0, \alpha \in(0,1), f \in C^{\alpha}([0, T] ; C[0,1])$,

$$
g_{j}, h_{j} \in C^{\alpha}([0, T] ; \mathbf{C}), \quad j=0,1 .
$$

For each $i, j=0,1$, let $\mathcal{A}_{i j}\left(\partial_{\xi}\right)$ (resp. $\mathcal{B}_{i j}\left(\partial_{\xi}\right)$ ) be a linear differential operator in $[0,1]$ with complex coefficients of the order not exceeding 3 (resp. of the order 1). We consider a damped Euler-Bernoulli beam equation with dynamic boundary 
conditions:

(4.12)

$$
\begin{cases}\partial_{t}^{2} u+\partial_{\xi}^{4} u-\rho \partial_{\xi}^{2} \partial_{t} u=f, & \text { in }(0, T] \times[0,1], \\ \partial_{t}^{2} u(t, j)+\mathcal{A}_{0 j}\left(\partial_{\xi}\right) u(t, j)+\mathcal{B}_{0 j}\left(\partial_{\xi}\right) \partial_{t} u(t, j)=g_{j}, & \text { in }(0, T] \times\{0,1\}, \\ \partial_{t}^{2} \partial_{\xi}^{2} u(t, j)+\mathcal{A}_{1 j}\left(\partial_{\xi}\right) u(t, j)+\mathcal{B}_{1 j}\left(\partial_{\xi}\right) \partial_{t} u(t, j)=h_{j}, & \text { in }(0, T] \times\{0,1\}, \\ u(0, \cdot)=\varphi_{0}, \quad \partial_{t} u(0, \cdot)=\varphi_{1}, & \text { in }[0,1], \\ \partial_{t} \partial_{\xi}^{2} u(0, j)=\psi_{j}, & j=0,1 .\end{cases}
$$

Take

$$
\begin{aligned}
& E=C[0,1], \quad X=\mathbf{C}^{4}, \\
& A=\frac{d^{4}}{d \xi^{4}} \quad \text { with } \quad \mathcal{D}(A)=C^{4}[0,1], \\
& B=-\rho \frac{d^{2}}{d \xi^{2}} \quad \text { with } \quad \mathcal{D}(B)=C^{2}[0,1], \\
& G_{0} \varphi=-\left(\begin{array}{l}
\mathcal{A}_{00}\left(\partial_{\xi}\right) \varphi(0) \\
\mathcal{A}_{01}\left(\partial_{\xi}\right) \varphi(1) \\
\mathcal{A}_{10}\left(\partial_{\xi}\right) \varphi(0) \\
\mathcal{A}_{11}\left(\partial_{\xi}\right) \varphi(1)
\end{array}\right) \quad \text { for } \quad \varphi \in \mathcal{D}\left(G_{0}\right):=\mathcal{D}(A) \\
& G_{1} \varphi=-\left(\begin{array}{l}
\mathcal{B}_{00}\left(\partial_{\xi}\right) \varphi(0) \\
\mathcal{B}_{01}\left(\partial_{\xi}\right) \varphi(1) \\
\mathcal{B}_{10}\left(\partial_{\xi}\right) \varphi(0) \\
\mathcal{B}_{11}\left(\partial_{\xi}\right) \varphi(1)
\end{array}\right) \quad \text { for } \quad \varphi \in \mathcal{D}\left(G_{1}\right):=\mathcal{D}(B) \\
& P \varphi=\left(\begin{array}{c}
\varphi(0) \\
\varphi(1) \\
\varphi^{\prime \prime}(0) \\
\varphi^{\prime \prime}(1)
\end{array}\right) \quad \text { for } \quad \varphi \in \mathcal{D}(P):=\mathcal{D}(A) \\
& P_{1} \varphi=\left\{\left(\begin{array}{c}
\varphi(0) \\
\varphi(1) \\
z_{3} \\
z_{4}
\end{array}\right) ; \quad z_{3}, z_{4} \in \mathbf{C}\right\} \quad \text { for } \quad \varphi \in \mathcal{D}\left(P_{1}\right):=\mathcal{D}(B), \\
& A_{1}=0, \quad B_{1}=0, \quad \widetilde{A}=0, \quad \widetilde{A}_{1}=0, \quad \widetilde{B}=0, \quad \widetilde{B}_{1}=0 .
\end{aligned}
$$

Then we have

$$
\begin{aligned}
& A_{0}=\frac{d^{4}}{d \xi^{4}} \text { with } \mathcal{D}\left(A_{0}\right)=\left\{\varphi \in C^{4}[0,1] ; \varphi(0)=\varphi(1)=\varphi^{\prime \prime}(0)=\varphi^{\prime \prime}(1)=0\right\}, \\
& B_{0}=-\rho \frac{d^{2}}{d \xi^{2}} \text { with } \mathcal{D}\left(B_{0}\right)=\left\{\varphi \in C^{2}[0,1] ; \varphi(0)=\varphi(1)=0\right\}, \\
& {[\mathcal{D}(A)]_{P} \simeq C^{4}[0,1], \quad[\mathcal{D}(B)]_{P_{1}} \simeq C^{2}[0,1] .}
\end{aligned}
$$


Obviously $\left(\mathrm{H}_{1}\right)$ and $\left(\mathrm{H}_{3}\right)$ are satisfied. So is $\left(\mathrm{H}_{2}\right)$ from [14, p. 1017 , line 4]. Furthermore, we know that $G_{0}$ (resp. $G_{1}$ ) is $A_{0}$-bounded (resp. $B_{0}$-bounded) with $A_{0}$-bound (resp. $B_{0}$-bound) zero (cf. [7] p. 170]). Thus the hypotheses of Theorem 2.3 are all satisfied. Therefore Theorem 3.3 is applicable. In this case,

$$
\begin{aligned}
& y(t)=\left(\begin{array}{c}
u(t) \\
x(t)
\end{array}\right), \quad u(t)=u(t, \cdot), \quad x(t):=\left(\begin{array}{c}
u(t, 0) \\
u(t, 1) \\
\partial_{\xi}^{2} u(t, 0) \\
\partial_{\xi}^{2} u(t, 1)
\end{array}\right), \quad t \in(0, T], \\
& \overline{\mathcal{D}(\mathbf{A}) \cap \mathcal{D}(\mathbf{B})}=\left\{\left(\begin{array}{l}
\varphi \\
x
\end{array}\right) \in C[0,1] \times \mathbf{C}^{4} ; \quad x \in P_{1} \varphi\right\}, \\
& \Upsilon \supset\left\{\left(\begin{array}{l}
\varphi \\
x
\end{array}\right) \in C^{2}[0,1] \times \mathbf{C}^{4} ; \quad P \varphi=x\right\} .
\end{aligned}
$$

It is not hard to verify (4.13). For 4.14), we exploit the fact (shown in the proof of [14 Theorem 5.1]) that

$$
\lim _{t \rightarrow 0^{+}} \inf \left\{t\|\psi\|_{C^{4}[0,1]}+\|\varphi-\psi\|_{C^{2}[0,1]}+t^{-1}\|\varphi-\psi\|_{C[0,1]} ; \quad \psi \in \mathcal{D}\left(A_{0}\right)\right\}=0
$$

for every

$$
\varphi \in \Omega:=\left\{\psi \in C^{2}[0,1] ; \quad \psi(0)=\psi(1)=\psi^{\prime \prime}(0)=\psi^{\prime \prime}(1)=0\right\} .
$$

Given $\varphi \in C^{2}[0,1]$, we put

$$
\varphi_{*}(\xi)=\varphi(0)+(\varphi(1)-\varphi(0)) \xi+\frac{1}{2} \varphi^{\prime \prime}(0) \xi^{2}+\frac{1}{6}\left(\varphi^{\prime \prime}(1)-\varphi^{\prime \prime}(0)\right) \xi^{3}, \quad \xi \in[0,1] .
$$

Then $\varphi-\varphi_{*} \in \Omega$. This in combination with (4.15) yields that

$$
\lim _{t \rightarrow 0^{+}} \Psi\left(t,\left(\begin{array}{c}
\varphi \\
P \varphi
\end{array}\right)\right)=0
$$

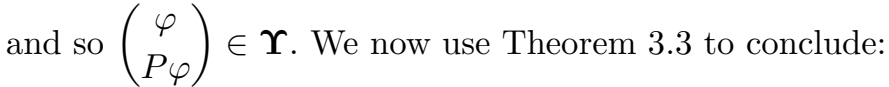

(i) For every $\varphi_{0} \in C^{2}[0,1], \varphi_{1} \in C^{1}[0,1], \psi_{j} \in \mathbf{C}(j=0,1)$, problem (4.12) has a unique solution

$$
u \in \bigcap_{i=0}^{2} C^{i}\left((0, T] ; C^{4-2 i}[0,1]\right) \bigcap\left(\bigcap_{k=0}^{1} C^{k}\left([0, T] ; C^{2-2 k}[0,1]\right)\right) .
$$

(ii) $\partial_{t}^{i} u \in C^{\alpha}\left([\varepsilon, T] ; C^{4-2 i}[0,1]\right)(\varepsilon \in(0, T), i=0,1,2)$ and

$$
\begin{aligned}
\|u(t, \cdot)\|_{C[0,1]} & \leq \mathrm{const}\left[\|f\|_{C([0, T] ; C[0,1])}+\sum_{j=0}^{1}\left(\left\|g_{j}\right\|_{C([0, T] ; \mathbf{C})}\right.\right. \\
& \left.\left.+\left\|h_{j}\right\|_{C([0, T] ; \mathbf{C})}+\left|\psi_{j}\right|\right)+\left\|\varphi_{0}\right\|_{C^{2}[0,1]}+\left\|\varphi_{1}\right\|_{C[0,1]}\right], \quad t \in[0, T] .
\end{aligned}
$$

(iii) If $\varphi_{0} \in C^{4}[0,1], \varphi_{1} \in C^{2}[0,1], \psi_{j}=\varphi_{1}^{\prime \prime}(j)(j=0,1)$, and

$$
\varphi_{0}^{(4)}(j)+\mathcal{A}_{0 j}\left(\partial_{\xi}\right) \varphi_{0}(j)-\rho \varphi_{1}^{\prime \prime}(j)+\mathcal{B}_{0 j}\left(\partial_{\xi}\right) \varphi_{1}(j)=f(0, j)-g_{j}, \quad j=0,1,
$$

then the solution $u$ belongs to $\bigcap_{i=0}^{2} C^{i}\left([0, T] ; C^{4-2 i}[0,1]\right)$. 
Here, for getting the uniqueness, the following fact was taken into account: if $u$ is a solution of problem (4.12) satisfying (4.16), then

$$
\begin{gathered}
x(t)=P u(t), \quad x^{\prime}(t)=P u^{\prime}(t), \quad t \in(0, T], \\
x(0)=\left(\begin{array}{l}
\varphi_{0}(0) \\
\varphi_{0}(1) \\
\varphi_{0}^{\prime \prime}(0) \\
\varphi_{0}^{\prime \prime}(1)
\end{array}\right), \quad x^{\prime}(0)=\left(\begin{array}{c}
\varphi_{1}(0) \\
\varphi_{1}(1) \\
\psi_{0} \\
\psi_{1}
\end{array}\right) .
\end{gathered}
$$

Remark 4.6. In the case of zero boundary value, i.e., when $\mathcal{A}_{i j}, \mathcal{B}_{i j}, g_{i}, h_{j}, \varphi_{i}(j)$, and $\psi_{j}(i, j=0,1)$ are all zero, conclusion (i) and a weaker form of conclusion (iii) are due to [14, Theorem 5.1].

\section{ACKNOWLEDGMENTS}

The authors would like to express their gratitude to Professors C. J. K. Batty, E. B. Davies and R. Nagel for their kind help and valuable suggestions. The authors would also like to thank Oxford University, King's College London, and Universität Tübingen for their hospitality.

\section{REFERENCES}

[1] K. T. Andrews, K. L. Kuttler, and M. Shillor, Second order evolution equations with dynamic boundary conditions, J. Math. Anal. Appl. 197 (1996), 781-795. MR 1373080 (96m:34116)

[2] H. T. Banks and D. J. Inman, On damping mechanisms in beams, ASME Trans. 58 (1991), 716-723.

[3] H. Brezis and L. E. Fraenkel, A function with prescribed initial derivatives in different Banach spaces, J. Funct. Anal. 29 (1978), 328-335. MR 0512249 (80k:46047)

[4] T. P. Chang, Forced vibration of a mass-loaded beam with a heavy tip body, J. Sound Vibration 164 (1993), 471-484.

[5] R. W. Carroll and R. E. Showalter, Singular and Degenerate Cauchy Problems, Academic Press, New York, 1976. MR 0460842 (57:834)

[6] V. Casarino, K. -J. Engel, R. Nagel and G. Nickel, A semigroup approach to boundary feedback systems, Integral Equations Operator Theory 47 (2003), 289-306. MR 2012840

[7] K. -J. Engel and R. Nagel, One-parameter Semigroups for Linear Evolution Equations, GTM 194, Springer, Berlin, New York, 2000. MR 1721989 (2000i:47075)

[8] J. Escher, Quasilinear parabolic systems with dynamical boundary conditions, Comm. Part. Diff. Equations 18 (1993), 1309-1364. MR 1233197 (94g:35112)

[9] H. O. Fattorini, The Cauchy Problem, Addison-Wesley, Reading, Mass. 1983. MR 0692768 (84g:34003)

[10] H. O. Fattorini, Second Order Linear Differential Equations in Banach Spaces, Elsevier Science Publishers B. V., Amsterdam, 1985. MR MR0797071 (87b:34001)

[11] A. Favini, G. R. Goldstein, J. A. Goldstein and S. Romanelli, $C_{0}$-semigroups generated by second order differential operators with general Wentzell boundary conditions, Proc. Amer. Math. Soc. 128 (2000), 1981-1989. MR 1695147 (2000m:47054)

[12] A. Favini, G. R. Goldstein, J. A. Goldstein and S. Romanelli, Generalized Wentzell boundary conditions and analytic semigroups in $C[0,1]$, Semigroups of operators: theory and applications (Newport Beach, CA, 1998), 125-131, Progr. Nonlinear Diff. Equations Appl., 42, Birkhäuser, Basel, 2000. MR 1788874 (2001h:47061)

[13] A. Favini, G. R. Goldstein, J. A. Goldstein and S. Romanelli, The heat equation with generalized Wentzell boundary condition, J. Evolution Eqs. 2 (2002), 1-19. MR 1890879 (2003b:35089)

[14] A. Favini and E. Obrecht, Conditions for parabolicity of second order abstract differential equations, Diff. Integral Equations, 4 (1991), 1005-1022. MR 1123349 (92m:47078)

[15] M. Grobbelaar-van Dalsen and N. Sauer, Dynamic boundary conditions for the Navier-Stokes equations, Proc. Roy. Soc. Edinburgh Sect. A 113 (1989), 1-11. MR 1025450 (91a:35136) 
[16] T. Hintermann, Evolution equations with dynamic boundary conditions, Proc. Roy. Soc. Edinburgh Sect. A 113 (1989), 43-60. MR 1025453 (91a:35094)

[17] J. Lagnese, Decay of solutions of wave equations in a bounded region with boundary dissipation, J. Diff. Equations 50 (1983), 163-182. MR 0719445 (85f:35025)

[18] J. L. Lions, Équations différentielles opérationelles et problèmes aux limites, Springer-Verlag, Berlin, 1961. MR 0153974 (27:3935)

[19] E. Obrecht, The Cauchy problem for time-dependent abstract parabolic equations of higher order, J. Math. Anal. Appl. 125 (1987), 508-530. MR 896179 (88j:34125)

[20] A. Pazy, Semigroups of Linear Operators and Applications to Partial Differential Equations, Springer-Verlag, New York, 1983. MR 0710486 (85g:47061)

[21] Y. Sakawa and Z. H. Luo, Modeling and control of coupled bending and torsional vibrations of flexible beams, IEEE Trans. Automat. Control 34 (1989), 970-977. MR 1007424 (90g:73087)

[22] R. E. Showalter, Degenerate evolution equations and applications, Indiana Univ. Math. J. 23 (1974), 655-677. MR 333835 (48:12157)

[23] H. Triebel, Interpolation Theory, Function Spaces, Differential Operators, North-Holland, Amsterdam, 1978. MR 0500580 (80i:46032a)

[24] T. J. Xiao and J. Liang, The Cauchy problem for higher order abstract differential equations, Lect. Notes in Math. 1701, Springer, Berlin, New York, 1998. MR 1725643 (2001a:34099)

[25] T. J. Xiao and J. Liang, Higher order abstract Cauchy problems and their existence, uniqueness families, J. London Math. Soc. 67 (2003), 149-164. MR 1942417 (2003i:34141)

Department of Mathematics, University of Science and Technology of China, Hefei, Anhui 230026, People's Republic of China - And - Mathematisches Institut, Universität Tübingen, Auf Der Morgenstelle 10, D-72076, Tübingen, Germany

E-mail address: xiaotj@ustc.edu.cn

E-mail address: tixi@fa.uni-tuebingen.de

Department of Mathematics, University of Science and Technology of China, Hefei, Anhui 230026, People's Republic of China - and - Mathematisches Institut, Universität Tübingen, Auf der Morgenstelle 10, D-72076, Tübingen, Germany

E-mail address: jliang@ustc.edu.cn

E-mail address: jili@fa.uni-tuebingen.de 